NASA Technical Memorandum 106584

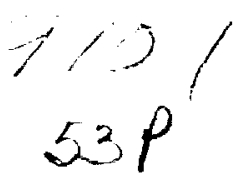

\title{
Interaction of Oblique Instability Waves With Weak Streamwise Vortices
}

M.E. Goldstein

National Aeronautics and Space Administration

Lewis Research Center

Cleveland, Ohio

and

David W. Wundrow

NYMA, Inc.

Engineering Services Division

Brook Park, Ohio

May 1994
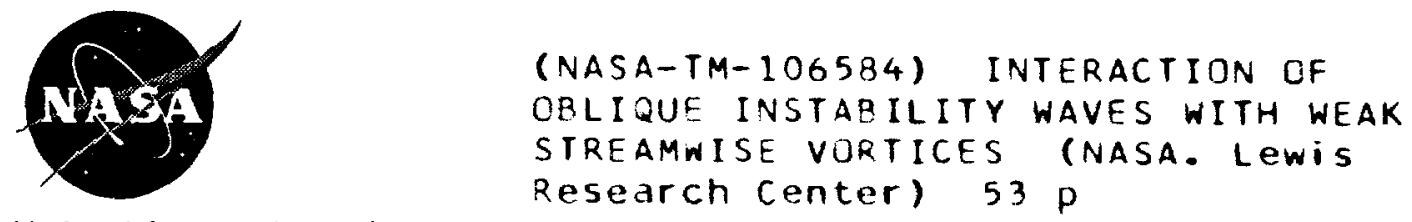

National Aeronautics and Space Administration 
INTERACTION OF OBLIQUE INSTABILITY WAVES WITH WEAK STREAMWISE VORTICES

M.E. Goldstein

National Aeronautics and Space Administration

Lewis Research Center

Cleveland, Ohio 44135

and

David W. Wundrow

NYMA, Inc.

Engineering Services Division

Brook Park, Ohio 44142

This paper is concerned with the effect of a weak spanwise-variable mean-flow distortion on the growth of oblique instability waves in a Blasius boundary layer. The streamwise component of the distortion velocity initially grows linearly with increasing streamwise distance, reaches a maximum, and eventually decays through the action of viscosity. This decay occurs slowly and allows the distortion to destabilize the Blasius flow over a relatively large streamwise region. It is shown that even relatively weak distortions can cause certain oblique Rayleigh instability waves to grow much faster than the usual two-dimensional Tollmien-Schlichting waves that would be the dominant instability modes in the absence of the distortion. The oblique instability waves can then become large enough to interact nonlinearly within a common critical layer. It is shown that the resulting nonlinearity is weak and that the common amplitude of the interacting oblique waves is governed by the amplitude evolution equation derived in Goldstein \& Choi (1989). The implications of these results for Klebanoff-type transition are discussed.

\section{Introduction}

Transition to turbulence in boundary layers usually begins with initially linear and noninteracting instability waves that grow to nonlinear amplitudes as they propagate down- 
stream. The first nonlinear stage of evolution - which might more appropriately be referred to as a modal interaction stage - is usually characterized by the rapid growth of three-dimensional disturbances due to resonant interactions between instability waves and between instability waves and streamwise vortices.

This phenomena is usually studied experimentally by exciting the flow with relatively two-dimensional single-frequency excitation devices. The initial motion, say just downstream of the excitation device, should then be periodic in time and reasonably well described by linear instability theory - provided, of course, the excitation levels are sufficiently small. The typical mean flow is relatively two-dimensional and fairly close to a Blasius profile at the low Mach numbers where most of the experiments have been carried out. The instability wave growth rates should then be small compared to the inverse of the mean boundary-layer thickness in these experiments.

When flow visualization devices are used, the initial modal interaction stage is evidenced by the appearance of $\Lambda$-shaped structures which can either be aligned or staggered in alternating rows. The aligned arrangement, which occurred in the original Klebanoff \& Tidstrom (1959) and Klebanoff, Tidstrom \& Sargent (1962) experiments, is usually referred to as 'peak-valley' splitting. It is believed to be a complex phenomena (Kachanov \& Levchenko $1984, \S 5.2$ ) that can be explained in terms of at least three different (relatively weak) resonant-type interaction mechanisms each of which probably plays a role in one or more of the many experiments that have been carried out to study this phenomena (Kachanov, Kozlov \& Levchenko 1985; Kachanov 1987; Hama \& Nutant 1963; Kovasznay, Komoda \& Vasudeva 1962; Nishioka, Asai \& lida 1979; and others).

A resonant-type interaction involving weak streamwise vortices seems to have played 
an important role in the original Klebanoff \& Tidstrom (1959) and Klebanoff et al. (1962) experiments. The present paper is an attempt to provide a systematic first-principles analysis of this phenomena by using high-Reynolds-number asymptotic methods. There has been a tendency in the literature to separate such analyzes into wave-wave and wave-vortex interactions. This paper, which brings together a number of recent ideas in order to shed some light on the Klebanoff-type transition process, turns out to be a combination both of these approaches.

We first consider the initial linear region, just downstream of the excitation device, where the instability waves are still small enough so that no significant modal interactions take place. The instability waves will grow on the relatively slow viscous time scale when the mean flow is two-dimensional, with the two-dimensional mode exhibiting the most rapid growth. However, even relatively weak spanwise-periodic mean-flow distortions (i.e. streamwise vortices) can cause certain oblique modes to grow on the inviscid time scale through a kind of resonant-interaction mechanism first considered for Görtler vortices by Nayfeh (1981) and later by Bennett \& Hall (1988), Nayfeh \& Al-Maaitah (1988) and Hall \& Seddougui (1989). This resonant interaction allows the oblique modes to grow faster than the plane wave once the Reynolds number becomes sufficiently large. The streamwise vortices, which are generated whenever quasi-periodic cross-flow velocities exist in the flow, have streamwise velocity components that initially grow in proportion to the downstream distance. These velocity components can then become quite large before viscous and/or nonlinear effects cause them to saturate ${ }^{1}$.

\footnotetext{
${ }^{1}$ This is related to, but somewhat different than, the algebraic growth mechanisms studied by Ellingsen \& Palm (1975), Hultgren \& Gustavsson (1981) and Landahl (1990)
} 
It is therefore appropriate to suppose that the spanwise-periodic motions are initiated by a steady cross flow with spanwise wavenumber, say $2 \beta$, and a pair of equal-amplitude oblique instability modes with the same streamwise wavenumber and scaled frequency but opposite spanwise wavenumbers, say $\pm \beta$. These two modes combine to form a standing wave in the spanwise direction that propagates only in the direction of the free stream. This situation is typical of wave excitation experiments which often involve relatively long excitation devices oriented perpendicular to the free-stream direction.

When the Reynolds number is sufficiently large, the streamwise vortices can persist over streamwise distances that are long enough to enable the oblique modes to reach nonlinear amplitudes. Since the oblique-mode growth rates turn out to be small compared to the spanwise wavenumbers in the present analysis, the initial nonlinear interactions are confined to a localized region centered around the so called 'critical level' where the streamwise component of the mean-flow velocity is equal to the common phase speed of the oblique modes. The flow outside the critical layer is still governed by linear dynamics and is given by the superposition of a Blasius flow, a spanwise-periodic mean-flow distortion and a pair of oblique instability modes. The common amplitude of the oblique modes is completely determined by the nonlinear dynamics within the critical layer.

The critical-layer nonlinearity turns out to be weak in the sense that it enters through an inhomogeneous term in a higher-order problem rather than through a coefficient in the lowest-order or dominant-balance equation. This ultimately means that the oblique-mode amplitude is completely determined by a single amplitude-evolution equation. It turns out that this equation is the same as the one that was obtained by Goldstein \& Choi (1989), who considered the related problem of the interaction of two oblique modes in a two-dimensional 
shear layer.

Since this type of interaction has already been shown to play a role in both the subharmonic transition process (Goldstein \& Lee 1992; Mankbadi, Wu \& Lee 1993; and Wundrow, Hultgren \& Goldstein 1994) as well as in one of the major competing scenarios for the harmonic transition process (Goldstein \& Lee 1992), this adds to the growing evidence that it is a relatively universal mechanism that can occur in many of the transition processes identified in the literature. It is our belief that this identification of universal mechanisms is one of the important ways in which the fundamental theory can contribute to our understanding of the very complex and multifaceted transition process.

The paper is organized as follows. The spanwise-periodic mean-flow distortion is analyzed in $\S 3$. The linear stability of this flow is considered in $\S \S 4$ and 5 where it is shown that even relatively small distortions can cause the oblique instability modes to grow more rapidly than the fastest growing plane wave when the Reynolds number is sufficiently large. The nonlinear effects are considered in $\S 6$ where it is shown that the nonlinearity is weak and the common amplitude of the oblique modes is governed by the amplitude evolutionequation derived in Goldstein \& Choi (1989), but with different numeric coefficients. The implications of the results are discussed in $§ 7$.

\section{Formulation}

To fix ideas, we consider the incompressible flow over an infinitely thin flat plate and suppose that a small-amplitude spanwise-periodic motion is suddenly imposed on the flow (say by a vibrating ribbon or other excitation device) at a distance $L$ downstream from the leading edge. The Cartesian coordinates system $(x, y, z)$ is attached to the plate at $L$ with 
$x$ aligned with the free stream, $y$ normal to the plate, and $z$ in the spanwise direction. All lengths are non-dimensionalized by $\delta$ where

$$
\delta \equiv L / R^{\frac{1}{2}}
$$

is characteristic of the mean boundary-layer thickness at $L$ and

$$
R \equiv L U_{\infty} / \nu
$$

is the global Reynolds number based on the free-stream velocity $U_{\infty}$ and the kinematic viscosity $\nu$. The time $t$, velocity $u=(u, v, w)$, and pressure variation $p$ from the freestream value $P_{\infty}$ are non-dimensionalized by $\delta / U_{\infty}, U_{\infty}$ and $\rho U_{\infty}^{2}$, respectively, where $\rho$ is the density. With this non-dimensionalization, the Navier-Stokes equations become

$$
\begin{gathered}
(\partial / \partial t+u \cdot \nabla) u+\nabla p=\nabla^{2} u / R^{\frac{1}{2}} \\
\nabla \cdot u=0
\end{gathered}
$$

where $\nabla \equiv i \partial / \partial x+j \partial / \partial y+k \partial / \partial z$ is the gradient operator.

When the excitation device is placed perpendicular to the free-stream direction, as we shall now assume, it will generate a disturbance that consists of a weak mean-flow distortion plus a pair of equal-amplitude oblique instability modes that form a standing wave in the spanwise direction. Since we ultimately require that the spanwise length scale of the disturbance, say $1 / \beta$, be much larger than the local boundary-layer thickness, we introduce the scale factor $\sigma$ through

$$
\sigma \bar{\beta} \equiv \delta \beta, \quad 0<\sigma \leqslant 1, \quad \bar{\beta}=O(1)
$$

and eventually require that $\sigma \ll 1$. The distinguished scaling for the imposed cross-flow velocity at $x=0$, say $\bar{\sigma} R^{-\frac{1}{2}} W_{0}(y, \sigma z)$ where $\bar{\sigma} \equiv 1 / \ln \sigma$, then corresponds to $W_{0}=O(1)$. 


\section{Mean-flow distortion}

The imposed cross flow $\bar{\sigma} R^{-\frac{1}{2}} W_{0}(y, \sigma z)$ generates a mean-flow distortion that ultimately decays out through the action of viscosity, but causes the mean flow to become inflectional before this occurs. This allows certain initially linear instabilities to grow to nonlinear amplitudes by essentially inviscid mechanisms. It turns out that the most inflectional profiles lie in the region where

$$
x_{2} \equiv x / \sigma^{3} R^{\frac{1}{2}}=O(1)
$$

for $0<\sigma \leqslant 1$. This scale will be very long compared to the triple-deck length scale when

$$
\sigma \gg 1 / R^{\frac{1}{3}}
$$

which is now assumed to be the case. When $\sigma=O(1)$, the mean-flow velocity $U=(U, V, W)$ and pressure $P$ expand like

$$
\begin{aligned}
U & =\left(\hat{U}, R^{-\frac{1}{2}} \hat{V}, R^{-\frac{1}{2}} \hat{W}\right)+\cdots \\
P & =R^{-1} \hat{P}+\cdots
\end{aligned}
$$

and are determined by the parabolized Navier-Stokes equations

$$
\begin{gathered}
\left(\hat{U} \partial / \partial x_{2}+\hat{U} \cdot \nabla_{T}\right) \hat{U}+\nabla_{T} \hat{P}=\nabla_{T}^{2} \hat{U} \\
\partial \hat{U} / \partial x_{2}+\nabla_{T} \cdot \hat{U}=0
\end{gathered}
$$

where $\hat{U} \equiv(\hat{U}, \hat{V}, \hat{W})$ and $\nabla_{T} \equiv j \partial / \partial y+k \partial / \partial z$ is the gradient in the transverse plane; but, when $\sigma \ll 1$, the mean flow will be interactive relative to the cross-flow direction and will be determined by the three-dimensional boundary-layer equations in the main part of the flow 
field. The initial distortion becomes fully interactive in a sufficiently small neighborhood of $x=0$, but this region is of little interest here since (with the present scaling) it has negligible effect on the instability waves. Its structure is discussed in appendix B for the long-wavelength limit $\sigma \ll 1$ in order to show how the $x_{2}$-scale flow (to be discussed below) evolves from the initial disturbance. Since (3.5) and (3.6) must be solved numerically, the relevant physical mechanisms can best be understood by concentrating on the longwavelength limit $\sigma \ll 1$ for which analytic solutions can be obtained. The discussion of the order-one-wavelength case is deferred to the end of $\S 7$. The structure of the long-wavelength solution is similar to the short-wavelength triple-deck solutions worked out by Rozhko \& Ruban (1987) and by Choudhari, Hall \& Streett (1992), but some new results are obtained.

\subsection{The main boundary layer}

In the main region where $x_{2}$ and $y$ are both order one, the spanwise-variable mean flow is an inviscid perturbation about the local Blasius profile $\left(U_{B}, R^{-\frac{1}{2}} V_{B}\right)$. The mean-flow velocity and pressure in this region expand like

$$
\begin{aligned}
U & =U_{B}+\sigma^{4} U_{D}+O\left(\sigma^{5}\right), \\
V & =R^{-\frac{1}{2}}\left[V_{B}+\sigma V_{D}+O\left(\sigma^{2}\right)\right], \\
W & =\bar{\sigma} R^{-\frac{1}{2}}\left[W_{D}+O(\sigma \ln \sigma)\right], \\
P & =\sigma^{-3} R^{-1}\left[P_{D}+O(\sigma)\right],
\end{aligned}
$$

where

$$
U_{B}\left(x_{2}, y\right)=f^{\prime}(y)-\sigma^{3} \frac{1}{2} x_{2} y f^{\prime \prime}(y), \quad V_{B}(y)=\frac{1}{2}\left[y f^{\prime}(y)-f(y)\right]
$$


$f$ denotes the Blasius function which satisfies

$$
f^{\prime \prime \prime}+\frac{1}{2} f f^{\prime \prime}=0, \quad f(0)=f^{\prime}(0)=0, \quad f^{\prime}(\infty)=1
$$

$U_{D}, V_{D}$, and $W_{D}$ are functions of $x_{2}, y$ and

$$
\bar{z} \equiv \sigma z
$$

$P_{D}$ is a function of $x_{2}$ and $\bar{z}$ only, and a prime denotes differentiation with respect to the argument. Substituting (3.7)-(3.10) into (2.3) and (2.4) and using the fact that

$$
W_{D} \rightarrow W_{0}(y, \bar{z}) \quad \text { as } \quad x_{2} \rightarrow 0
$$

leads to

$$
\begin{aligned}
U_{D} & =f^{\prime \prime}\left(\mathcal{A}+\bar{\sigma} x_{2} H_{\bar{z}}\right), \\
V_{D} & =-f^{\prime}\left(\mathcal{A}_{x_{2}}+\bar{\sigma} H_{\bar{z}}\right), \\
W_{D} & =W_{0},
\end{aligned}
$$

where

$$
H(y, \bar{z}) \equiv \int_{0}^{y}\left[\frac{W_{0}(s, \bar{z})}{f^{\prime}(s)}-\frac{W_{0}(0, \bar{z})}{\lambda_{0} s}\right] \mathrm{d} s+\frac{W_{0}(0, \bar{z})}{\lambda_{0}} \ln y
$$

$\lambda_{0} \approx 0.33206$ is the Blasius wall-shear stress and an independent variable used as a subscript denotes differentiation with respect to that variable. The particle displacement $\mathcal{A}$ and pressure distribution $P_{D}$ are, at this point, arbitrary functions of $x_{2}$ and $\bar{z}$, which have the implicit $\sigma$ dependence

$$
\mathcal{A}=\mathcal{A}_{0}\left(x_{2}, \bar{z}\right)+\bar{\sigma} \mathcal{A}_{1}\left(x_{2}, \bar{z}\right), \quad P_{D}=P_{0}\left(x_{2}, \bar{z}\right)+\bar{\sigma} P_{1}\left(x_{2}, \bar{z}\right),
$$


where the $O(1)$ terms have been inserted to facilitate matching with the solution in the viscous wall layer to be discussed below. We do, however, require that

$$
\mathcal{A}, \mathcal{A}_{x_{2}} \rightarrow 0 \text { as } \quad x_{2} \rightarrow 0
$$

so the velocity distortions $U_{D}$ and $V_{D}$ arise from only the imposed cross flow $W_{0}$. A $\sigma$ dependence similar to that in $P_{D}$ will be implicitly assumed in the corresponding dependent variables in the wall layer as well as in the inviscid outer layer which is considered next.

\subsection{The outer layer}

Since the solution (3.16) does not vanish as $y \rightarrow \infty$, it is necessary to introduce an outer region where

$$
\bar{y} \equiv \sigma y=O(1)
$$

in order to satisfy the appropriate free-stream boundary conditions. The solution in this region relates $P_{D}$ and $\mathcal{A}$.

Substituting (3.1), (3.13) and (3.21) into (2.3) and (2.4) and matching with the mainboundary-layer flow shows that the solution in the outer layer expands like

$$
\begin{aligned}
U & =1+\sigma^{-3} R^{-1} \bar{U}+\cdots \\
V & =R^{-\frac{1}{2}} V_{B}(\infty)+\sigma R^{-\frac{1}{2}} \bar{V}+\cdots \\
W & =\sigma R^{-\frac{1}{2}} \bar{W}+\cdots \\
P & =\sigma^{-3} R^{-1} \bar{P}+\cdots
\end{aligned}
$$

where it has been assumed that $W_{0}=o\left(y^{-1}\right)$ as $y \rightarrow \infty$. The functions $\bar{U}, \bar{V}, \bar{W}$ and $\bar{P}$ of $x_{2}, \bar{y}$ and $\bar{z}$ are determined by the linearized Euler equations. It follows from these 
equations that

$$
\vec{P}_{\bar{y} \bar{y}}+\bar{P}_{\bar{z} \bar{z}}=0
$$

while the free-stream boundary conditions and matching with the main-boundary-layer solution require that

$$
\bar{P} \rightarrow \mathbf{0} \text { as } \bar{y} \rightarrow \infty
$$

and

$$
\bar{P}=P_{D}, \quad \bar{P}_{\bar{y}}=\mathcal{A}_{x_{2} x_{2}} \quad \text { at } \quad \bar{y}=0 .
$$

The solution to (3.26)-(3.28) is most easily found by expressing $\bar{P}$ as the real part of an analytic function of the complex variable $\bar{z}+\mathrm{i} \bar{y}$. The Cauchy integral formula can then be used to relate $P_{D}$ and $\mathcal{A}$. For the spanwise-periodic mean flows that are of interest here, this leads to

$$
P_{D_{\bar{z}}}=\frac{\bar{\beta}}{\pi} f_{0}^{\pi / \bar{\beta}} \mathcal{A}_{x_{2} x_{2}}\left(x_{2}, \zeta ; \sigma\right) \cot \bar{\beta}(\bar{z}-\zeta) \mathrm{d} \zeta,
$$

where $f$ denotes the Cauchy principal value.

\subsection{The viscous wall layer}

It will now be assumed that

$$
B(\bar{z}) \equiv W_{0}(0, \bar{z}) \neq 0 .
$$

(The case where $W_{0}(0, \bar{z})=0$ is much more complex and is best studied by considering the order-one-wavelength problem discussed at the end of $\S 7$.$) It then follows from (3.15),$ (3.18) and (3.30) that

$$
U_{D} \rightarrow \lambda_{0} \mathcal{A}+\bar{\sigma} x_{2} B^{\prime} \ln y \quad \text { as } \quad y \rightarrow 0
$$


and consequently that $U$ will not satisfy the proper wall boundary condition. It is therefore necessary to introduce a viscous wall layer where

$$
Y=y / \sigma=O(1)
$$

in order to bring $U$ to zero at the wall. The mean velocity in this region expands like

$$
\begin{aligned}
U & =\sigma \lambda_{0} Y+\sigma^{4}\left(\tilde{U}-\frac{1}{48} \lambda_{0}^{2} Y^{4}-\frac{1}{2} \lambda_{0} x_{2} Y\right)+\cdots, \\
V & =\sigma^{2} R^{-\frac{1}{2}}\left(\tilde{V}+\frac{1}{4} \lambda_{0} Y^{2}\right)+\cdots \\
W & =R^{-\frac{1}{2}} \tilde{W}+\cdots
\end{aligned}
$$

where $\tilde{U}, \tilde{V}$ and $\tilde{W}$ are functions of $x_{2}, Y$ and $\bar{z}$.

Substituting (3.33)-(3.35) and (3.10) into (2.3) and (2.4) yields

$$
\begin{gathered}
\lambda_{0} Y \tilde{U}_{x_{2}}+\lambda_{0} \tilde{V}=\tilde{U}_{Y Y}, \\
\lambda_{0} Y \tilde{W}_{x_{2}}+P_{D_{\bar{z}}}=\tilde{W}_{Y Y}, \\
\tilde{U}_{x_{2}}+\tilde{V}_{Y}+\tilde{W}_{\bar{z}}=0 .
\end{gathered}
$$

It follows from (3.30) and (3.31) that the solution to these equations must satisfy the boundary conditions

$$
\tilde{U}=\tilde{V}=\tilde{W}=0 \quad \text { at } \quad Y=0
$$

and

$$
\tilde{U} \rightarrow \lambda_{0} \mathcal{A}+\bar{\sigma} x_{2} B^{\prime} \ln (\sigma Y), \quad \tilde{W} \rightarrow \bar{\sigma} B \quad \text { as } \quad Y \rightarrow \infty
$$

The solution must also satisfy the upstream condition

$$
\tilde{U} \rightarrow 0, \quad \tilde{W} \rightarrow \bar{\sigma} B \quad \text { as } \quad x_{2} \rightarrow 0
$$


since the thickness of the viscous wall layer goes to zero as $x_{2} \rightarrow 0$.

Eliminating $\tilde{V}$ between (3.36) and (3.38) leads to

$$
\lambda_{0} Y \tilde{U}_{Y x_{2}}-\lambda_{0} \tilde{W}_{\bar{z}}=\tilde{U}_{Y Y Y}
$$

which determines $\tilde{U}$ once $\tilde{W}$ is known. This equation must be solved subject to

$$
\tilde{U}_{Y Y}=0 \text { at } \quad Y=0
$$

in addition to (3.39)-(3.41). Since the boundary-value problem (3.37) and (3.39)-(3.43) has a similarity solution when $P_{D}$ is set equal to zero and $\mathcal{A}$ is selected appropriately, it is convenient to seek a solution of the form

$$
\tilde{U}=\bar{\sigma} x_{2} B^{\prime}(\bar{z}) F(\eta)+\tilde{U}^{(I)}\left(x_{2}, Y, \bar{z} ; \sigma\right), \quad \tilde{W}=\bar{\sigma} B(\bar{z}) G(\eta)+\tilde{W}^{(I)}\left(x_{2}, Y, \bar{z} ; \sigma\right)
$$

where

$$
\eta \equiv\left(\lambda_{0} / x_{2}\right)^{\frac{1}{3}} Y
$$

and $F$ and $G$ are determined by

$$
F^{\prime \prime \prime}+\frac{1}{3} \eta^{2} F^{\prime \prime}-\frac{2}{3} \eta F^{\prime}=-G, \quad G^{\prime \prime}+\frac{1}{3} \eta^{2} G^{\prime}=0
$$

with

$$
F=F^{\prime \prime}=G=0 \quad \text { at } \quad \eta=0,
$$

and

$$
F \rightarrow \ln \eta, \quad G \rightarrow 1 \quad \text { as } \quad \eta \rightarrow \infty .
$$

It follows that

$$
G=\Gamma\left(\frac{1}{3}\right)^{-1} \gamma\left(\frac{1}{3}, \tau\right),
$$


where $\tau \equiv \eta^{3} / 9, \Gamma$ is the usual gamma function and $\gamma$ is the incomplete gamma function defined on page 260 of Abramowitz \& Stegun (1964). It can also be shown that

$$
F=\frac{1}{2} \Gamma\left(\frac{1}{3}\right) \Gamma\left(\frac{2}{3}\right)^{-1} \int_{0}^{\tau} t^{-\frac{2}{3}} \mathrm{e}^{-t} \mathrm{M}\left(\frac{4}{3}, \frac{2}{3}, t\right) \mathrm{d} t-\frac{3}{2} \Gamma\left(\frac{1}{3}\right)^{-1}\left[\tau^{\frac{1}{3}} \mathrm{e}^{-\tau}+\left(\tau+\frac{2}{3}\right) \gamma\left(\frac{1}{3}, \tau\right)\right]
$$

where $M$ denotes the confluent hypergeometric function in the notation of Abramowitz \& Stegun (1964, p. 504).

The inhomogeneous solutions $\tilde{U}^{(I)}$ and $\tilde{W}^{(I)}$ can be found by taking Laplace transforms with respect to $x_{2}$. The details are given in appendix $A$ where it is shown that

$$
\chi P_{D_{\bar{z} \bar{z}}}=-\Gamma\left(\frac{1}{3}\right) Q_{\bar{z}}^{(1)}-\int_{0}^{x_{2}} \mathcal{A}_{\xi \xi}(\xi, \bar{z} ; \sigma)\left(x_{2}-\xi\right)^{-\frac{2}{3}} \mathrm{~d} \xi
$$

and

$$
\lambda_{0}^{\frac{2}{3}} \chi \tilde{U}^{(I)}=-\int_{0}^{x_{2}}\left[Q_{\xi \bar{z}}^{(6)}(\xi, \bar{z} ; \sigma)+\mathcal{A}_{\xi}(\xi, \bar{z} ; \sigma)\right] F^{(I)}\left(\lambda_{0}^{\frac{1}{3}}\left(x_{2}-\xi\right)^{-\frac{1}{3}} Y\right) \mathrm{d} \xi
$$

where $\chi \equiv \Gamma\left(\frac{1}{3}\right)^{2} /\left(9 \lambda_{0}^{5}\right)^{\frac{1}{3}}$,

$$
Q^{(m)}\left(x_{2}, \bar{z} ; \sigma\right) \equiv \frac{\bar{\sigma} x_{2}^{\frac{\pi}{3}-1} B(\bar{z})}{\lambda_{0} \Gamma\left(\frac{m}{3}\right)}\left\{\ln \left[\sigma\left(\frac{x_{2}}{\lambda_{0}}\right)^{\frac{1}{3}}\right]-C_{1}+\frac{1}{3}\left[\psi(2)-\psi\left(\frac{m}{3}\right)\right]\right\}
$$

$F^{(I)}$ is given by (A 17), $C_{1}$ is given by (A 7$)$ and $\psi$ denotes the digamma function defined on page 258 of Abramowitz \& Stegun (1964).

The pressure distribution $P_{D}$ and the particle displacement $\mathcal{A}$ can now be found from the coupled equations (3.29) and (3.51). These equations, together with (3.15), (3.16) and (3.20), imply that

$$
U_{D} \sim \bar{\sigma} x_{2} f^{\prime \prime} H_{\bar{z}} \quad \text { and } \quad V_{D} \sim-\bar{\sigma} f^{\prime} H_{\bar{z}} \quad \text { as } \quad x_{2} \rightarrow 0
$$

while

$$
P_{D_{\bar{z}}} \sim-\frac{\bar{\sigma} B}{\lambda_{0} \chi x_{2}^{\frac{2}{3}}}\left\{\ln \left[\sigma\left(\frac{x_{2}}{\lambda_{0}}\right)^{\frac{1}{3}}\right]-C_{1}+\frac{1}{3}\left[\psi(2)-\psi\left(\frac{1}{3}\right)\right]\right\} \quad \text { as } \quad x_{2} \rightarrow 0
$$


which shows that the expansions (3.7)-(3.10) become invalid (as already anticipated) when $x_{2}$ becomes sufficiently small, i.e. when

$$
\hat{x} \equiv \sigma x=O(1)
$$

The relevant solution is constructed in appendix $B$ where it is shown that the pressure fluctuation that matches onto (3.55) as $\hat{x} \rightarrow \infty$ remains bounded at $\hat{x}=0$.

Equations (3.20), (3.29) and (3.51) also imply that

$$
\mathcal{A} \sim-\frac{\bar{\sigma} x_{2} B^{\prime}}{\lambda_{0}}\left\{\ln \left[\sigma\left(\frac{x_{2}}{\lambda_{0}}\right)^{\frac{1}{3}}\right]-C_{1}\right\} \quad \text { as } \quad x_{2} \rightarrow \infty,
$$

which, when combined with (3.15) and (3.45), shows that the viscous wall-layer thickness increases like $x_{2}^{\frac{1}{3}}$ as $x_{2} \rightarrow \infty$ and that it fills the entire Blasius boundary layer when $x / R^{\frac{1}{2}}=\sigma^{3} x_{2}=O(1)$. However, (3.7) implies that the streamwise velocity component of the vortex flow will still be small compared to the Blasius profile in this region. This, in turn, shows that the vortices undergo their entire life cycle from initial algebraic growth to ultimate viscous decay while still remaining small compared to the basic Blasius flow.

\section{Structure of the instability waves}

We now consider the oblique instability modes that are generated by the excitation device and which initially grow in accordance with linear dynamics. If it were not for the streamwise vortices, only the slowly growing Tollmien-Schlichting waves would be amplified by the mean flow but, as noted by Prandtl (1935) and Stuart (1965), the rapidly growing streamwise velocity perturbation can cause the mean flow to become inflectional and thereby support inviscid Rayleigh instabilities that exhibit much larger growth rates (when $\sigma$ is sufficiently large relative to $R^{-1}$ ). The inflection point will always lie close to the wall 
since the vortex velocity is always small compared to $U_{B}$ (the actual distance turns out to be $O(\sigma)$ ). The maximum inviscid growth of the instability waves will take place in the streamwise region where $x_{2}=O(1)$ since it follows from (3.44), (3.50), (3.52) and (3.57) that

$$
\tilde{U}_{Y Y} \rightarrow 0 \text { as } x_{2} \rightarrow \infty \text { at } Y=\text { constant }
$$

The expansions (3.7)-(3.9) and (3.33)-(3.35) and the inequality (3.2) show that the cross-flow velocity components of the vortex remain small compared to its streamwise component in this region. This means that the initially linear instability modes, whose wavelengths are small on the $x_{2}$ scale, are only affected by this latter velocity component and therefore behave (to lowest order of approximation) like linear perturbations of the unidirectional transversely sheared mean flow $U\left(x_{2}, y, \bar{z} ; \sigma\right)$, with the slow streamwise variable $x_{2}$ entering only parametrically, i.e. playing the role of a constant. It therefore follows from inviscid instability theory that the wavelength of the most rapidly growing waves will be $O\left(\delta \sigma^{-1}\right)$ which is long compared to the boundary-layer thickness but small compared to the downstream distance from the excitation device, say $x=\sigma^{3} R^{\frac{1}{2}} \ell$ where $\ell$ is order one.

The initially linear non-interacting instability waves will eventually become nonlinear when their amplitudes become sufficiently large. However, the resulting nonlinear effects will be confined to the critical layer since the linear growth rates are small compared to the corresponding wavenumbers and the Reynolds number is large.

The flow outside this layer will still be determined by linear dynamics. Its velocity and pressure will expand like

$$
\boldsymbol{u}=U+\epsilon(\dot{u}, \sigma \dot{v}, \dot{w})+\cdots
$$




$$
p=P+\epsilon \dot{p}+\cdots,
$$

where $\dot{u}, \dot{v}, \dot{w}$, and $\dot{p}$ are functions of

$$
\bar{x} \equiv \sigma x-\sigma^{4} R^{\frac{1}{2}} \ell,
$$

$y, \bar{z}$ and

$$
\bar{t} \equiv \sigma t
$$

and $\epsilon \ll 1$ characterizes the local amplitude of the unsteady disturbance in the streamwise region where nonlinear effects first become important. The precise relationship between $\epsilon$ and $\sigma$ will be specified below when the flow in the critical layer is considered.

Substituting (4.2) and (4.3) into (2.3) and (2.4) and linearizing the result about the mean flow $U$ yields

$$
\begin{gathered}
\mathrm{D}(\dot{u}, \dot{v}, \dot{w})+\left(U_{y} \dot{v}+U_{\bar{z}} \dot{w}, 0,0\right)+\left(\dot{p}_{\bar{x}}, \sigma^{-2} \dot{p}_{y}, \dot{p}_{\bar{z}}\right)=O\left(\sigma^{-1} R^{-\frac{1}{2}}\right), \\
\dot{u}_{\bar{x}}+\dot{v}_{y}+\dot{w}_{\bar{z}}=0,
\end{gathered}
$$

where $\mathrm{D} \equiv \partial / \partial \bar{t}+U \partial / \partial \bar{x}$ is the leading-order convective derivative relative to the mean flow. These equations are just the familiar equations for the linear perturbations about a uni-directional transversely sheared mean flow (Goldstein 1976; Henningson 1987). It is well known that the velocity fluctuations can be eliminated between them (see Goldstein 1976, pp. 6-10 for a detailed derivation) to obtain the following equation for the pressure fluctuation

$$
\mathrm{D}\left(\sigma^{-2} \dot{p}_{y y}+\nabla_{H}^{2} \dot{p}\right)-\sigma^{-2} 2 U_{y} \dot{p}_{\bar{x} y}-2 U_{\bar{z}} \dot{p}_{\bar{x} \bar{z}}=O\left(\sigma^{-1} R^{-\frac{1}{2}}\right)
$$

where $\nabla_{H}^{2} \equiv \partial^{2} / \partial \bar{x}^{2}+\partial^{2} / \partial \bar{z}^{2}$ is the Laplacian in the horizontal plane. 
Since $\sigma \ll 1$, our interest is in the long-wavelength instability wave solutions to (4.8). We subsequently show that, as in the case of a strictly two-dimensional mean flow, the relevant $x$-scale growth rates are $O\left(\sigma^{4}\right)$, which means that the solutions grow on the relatively long scale

$$
x_{1} \equiv \sigma^{4}\left(x-\sigma^{3} R^{\frac{1}{2}} \ell\right)=\sigma^{3} \bar{x} .
$$

As a minimum, we want $\sigma$ to be large enough so that these growth rates are at least as large as the relatively small, i.e. $O\left(R^{-\frac{1}{5}}\right)$, viscous growth rates corresponding to the upperbranch scaling for the Tollmien-Schlichting waves (Bodonyi \& Smith 1981; Goldstein \& Durbin 1986). This means that we should require

$$
R^{-\frac{1}{20}}=O(\sigma)
$$

which is consistent with the inequality (3.2) and includes the case $R^{-\frac{1}{20}}=o(\sigma)$ in which the growth rates are larger than the corresponding upper-branch growth rates of the TollmienSchlichting waves. In any event, the $O\left(\sigma^{-1} R^{-\frac{1}{2}}\right)$ error terms will then be small compared to the wave-growth terms in (4.6)-(4.8) and these equations will then be accurate enough to determine the instability wave solutions outside the critical layer - provided we allow for a thin Stokes layer in the near wall region where $y=O\left(\sigma^{-1} R^{-\frac{1}{4}}\right)$.

As already indicated, the initial upstream disturbance is best represented by a pair of equal-amplitude oblique instability modes having the same streamwise wavenumber and scaled frequency but opposite spanwise wavenumbers. These modes form a standing wave in the spanwise direction that exhibits its most rapid growth when its spanwise wavelength is twice that of the mean-flow distortion, i.e. when its spanwise wavelength is $2 \pi / \bar{\beta}$.

The resulting solution for the unsteady portion of the flow will then be of the form 
originally assumed by Henningson (1987), namely

$$
(\dot{u}, \hat{v}, \hat{w}, \dot{p})=\operatorname{Re}\left[A^{\dagger}(\hat{u}, \hat{v}, \hat{w}, \hat{p}) \mathrm{e}^{\mathrm{i} X}+B^{\dagger}(\hat{g}, 0,0,0)\right]
$$

where $A^{\dagger}\left(x_{1}\right)$ is a slowly varying amplitude function that accounts for the growth of the instability waves, $\operatorname{Re}\left[B^{\dagger}\left(x_{1}\right) \hat{g}(y, \bar{z})\right]$ is a spanwise-variable mean-flow distortion that is generated by nonlinear effects in the critical layer,

$$
X \equiv \bar{\alpha}(\bar{x}-\sigma \bar{c} \bar{t})
$$

and the real quantities $\bar{\alpha}(\sigma)$ and $\bar{c}(\sigma)$ are the scaled streamwise wavenumber and phase speed correct up to but not including $O\left(\sigma^{3}\right)$ terms. $\bar{\alpha}$ and $\bar{c}$ possess expansions of the form

$$
\bar{\alpha}=\bar{\alpha}_{0}+\cdots, \quad \bar{c}=\bar{c}_{0}+\cdots
$$

as $\sigma \rightarrow 0$ where $\bar{\alpha}_{0}$ and $\bar{c}_{0}$ are order-one constants. The corresponding scaled Strouhal number or (non-dimensional) angular frequency is

$$
\bar{S}=\bar{\alpha} \bar{c}
$$

where $\bar{S}$ is an order-one real constant. Substituting (4.11) into (4.8) shows that, outside the Stokes layer, the function $\hat{p}$ of $x_{1}, y$ and $\bar{z}$ is determined to the required order of accuracy by

$$
\left[\frac{\hat{p}_{y}}{(U-c)^{2}}\right]_{y}+\left[\frac{\sigma^{2} \hat{p}_{\bar{z}}}{(U-c)^{2}}\right]_{\bar{z}}-\frac{\sigma^{2} \alpha^{2} \hat{p}}{(U-c)^{2}}=0
$$

where

$$
\alpha \equiv \bar{\alpha}-\sigma^{3} \mathrm{i} A^{\dagger^{\prime}} / A^{\dagger} \text { and } c \equiv \sigma \bar{S} / \alpha
$$

which insures that the instability wave exhibits only spatial growth. It follows from (4.6), (4.7) and (4.11) that the velocity fluctuations are determined in terms of $\hat{p}$ by

$$
\hat{u}=-\frac{U_{y} \hat{p}_{y}}{\sigma^{2} \alpha^{2}(U-c)^{2}}-\frac{U_{\bar{z}} \hat{p}_{\bar{z}}}{\alpha^{2}(U-c)^{2}}-\frac{\hat{p}}{U-c}
$$




$$
\begin{gathered}
\hat{v}=\frac{\mathrm{i} \hat{p}_{y}}{\sigma^{2} \alpha(U-c)}, \\
\hat{w}=\frac{\mathrm{i} \hat{p}_{\bar{z}}}{\alpha(U-c)} .
\end{gathered}
$$

The solution to (4.15) that satisfies the homogeneous boundary condition

$$
\hat{p} \rightarrow 0 \text { as } y \rightarrow \infty
$$

and matches onto the Stokes-layer solution as $y \rightarrow 0$ is derived in the following section.

\section{Solution outside the critical layer}

\subsection{The Stokes layer}

Viscous effects, if they enter the reckoning, can only affect the solution within the critical layer and within a thin Stokes layer induced by the no-slip boundary condition at the wall. The appropriate scaled transverse variable for the latter region is

$$
\bar{Y}=\sigma R^{\frac{1}{2}} y .
$$

Substituting (4.2), (4.11) and (5.1) into (2.3) and (2.4) shows that the unsteady flow is determined to the required order of approximation in this region by

$$
\begin{gathered}
-\mathrm{i} \sigma \bar{S}\left(\hat{u}, \sigma R^{\frac{1}{v}} \hat{v}, \hat{w}\right)+\left(\mathrm{i} \bar{\alpha} \hat{p}, R^{\frac{1}{2}} \hat{p}_{\bar{Y}}, \hat{p}_{\bar{z}}\right)=\sigma\left(\hat{u}, \sigma R^{\dagger} \hat{v}, \hat{w}\right)_{\bar{Y} \bar{Y}}, \\
\mathrm{i} \bar{\alpha} \hat{u}+\sigma R^{\frac{1}{4}} \hat{v}_{\bar{Y}}+\hat{w}_{\bar{z}}=0
\end{gathered}
$$

together with the no-slip boundary condition

$$
\hat{u}=\hat{v}=\hat{w}=0 \quad \text { at } \quad \bar{Y}=0 .
$$

The velocity fluctuations can be eliminated between (5.2) and (5.3) to arrive at an equation for the pressure fluctuation. The equation for the transverse velocity fluctuation $\hat{v}$ can then 
be obtained by combining the $\vec{Y}$ derivative of this equation with the transverse component of (5.2). The solution that satisfies (5.4) and does not exhibit exponential growth as $\bar{Y} \rightarrow \infty$ is

$$
\hat{v}=\sigma^{-1} R^{-\frac{1}{4}} a \bar{\omega}^{-3}\left(\bar{\omega} \bar{Y}+\mathrm{e}^{-\bar{\omega} \bar{Y}}-1\right)
$$

where $\bar{\omega} \equiv \mathrm{e}^{-\mathrm{i} \frac{\pi}{T}} \bar{S}^{\frac{1}{2}}$ and $a$ is an arbitrary function of $x_{1}$ and $\bar{z}$. It follows from (5.5) and the transverse component of $(5.2)$ that

$$
\hat{p}_{\bar{Y}}=\sigma R^{-\frac{1}{2}} a\left(\bar{Y}-\bar{\omega}^{-1}\right)
$$

which now can be used to derive the appropriate boundary condition for (4.15) as $y \rightarrow 0$.

\subsection{The inviscid wall layer}

Introducing the wall-layer variable (3.32) into (4.15) and (5.6) and integrating the former with respect to $Y$ subject to matching with the latter as $Y \rightarrow 0$ shows that

$$
\hat{p}=\sigma b+O\left(\sigma^{5}\right)
$$

for $Y=O(1)$ where $b$ is an arbitrary function of $x_{1}$ and $\bar{z}$ that has an expansion in $\sigma$ containing terms up to but not including $O\left(\sigma^{4}\right)$. It turns out that

$$
b=b_{0}+\cdots+\mathrm{i} \sigma^{3} b_{3 i}+\cdots
$$

as $\sigma \rightarrow 0$ where $b_{0}$ depends on $\bar{z}$ only and the coefficients $b_{0}$ to $b_{3 i}$ are purely real so that $b_{0}$ and $\sigma^{3} b_{3 i}$ are the leading-order approximations to the real and imaginary parts of $b$.

To determine the dispersion relation, the small- $\sigma$ expansion of $\hat{p}_{Y}$ is needed. By using (5.7) together with the expansions (3.33) and (4.16), (4.15) can be integrated with respect 
to $Y$ to obtain, after some manipulation,

$$
\begin{aligned}
\frac{\hat{p}_{Y}}{(U-\sigma \bar{c})^{2}} & =\sigma^{3} \mathcal{D}\left[\frac{Y}{\lambda_{0}^{2} Y_{c}\left(Y-Y_{c}\right)}\right] \\
+\frac{\sigma^{6}}{\lambda_{0}^{3}} \mathcal{D} & {\left[\Phi+\bar{\mu}\left(\phi^{ \pm}-\phi^{-}\right)-\frac{\bar{c} Y^{2}}{\bar{\alpha} Y_{c}^{2}\left(Y-Y_{c}\right)^{2}} \frac{\mathrm{i} A^{\dagger^{\prime}}}{A^{\dagger}}+\frac{\lambda_{0}^{3}}{\sigma^{5} R^{\dagger} \bar{c}^{2} \bar{\omega}}\right]+\cdots }
\end{aligned}
$$

where $\mathcal{D} \equiv b_{\bar{z}} \partial / \partial \bar{z}+b_{\bar{z} \bar{z}}-\alpha^{2} b$, the transverse position of the critical level $y_{c}=\sigma Y_{c}$ is determined by the condition

$$
U\left(\ell, y_{c}, \bar{z} ; \sigma\right)=\sigma \bar{c}
$$

the \pm superscript indicates different values for $Y \geqslant Y_{c}, \Phi$ is the real function of $Y$ and $\bar{z}$ determined by

$$
\frac{1}{2}\left(Y-Y_{c}\right)^{3} \Phi_{Y}=\tilde{U}(\ell, Y, \bar{z} ; \sigma)-\tilde{U}\left(\ell, Y_{c}, \bar{z} ; \sigma\right)-\frac{1}{48} \lambda_{0}^{2}\left(Y^{4}-Y_{c}^{4}\right)-\frac{1}{2} \lambda_{0} \ell\left(Y-Y_{c}\right)
$$

with $\Phi=0$ at $Y=0$, and

$$
\bar{\mu}=\tilde{U}_{Y Y}\left(\ell, Y_{c}, \bar{z} ; \sigma\right)-\frac{1}{4} \lambda_{0}^{2} Y_{c}^{2}
$$

is the normal derivative of the scaled mean vorticity at the critical level. Matching (5.9) with the Stokes-layer solution (5.6) as $Y \rightarrow 0$ shows that

$$
a=-b_{\bar{z} \bar{z}}+\alpha^{2} b
$$

where the small- $\sigma$ expansion

$$
Y_{c}=\bar{c} / \lambda_{0}+O\left(\sigma^{3}\right)
$$

which is easily obtained from (3.7), (3.11) and (5.10), has been used.

It follows from (4.17) and (5.7) that the discontinuity in (5.9) results in a jump in the streamwise component of the velocity fluctuation

$$
\Delta \hat{u}=-\sigma^{3} \mathcal{D}\left[\bar{\mu}\left(\phi^{+}-\phi^{-}\right) / \lambda_{0}^{2} \bar{\alpha}^{2}\right]+\cdots
$$


across the critical layer. Matching this jump with the one induced by the flow in the critical layer determines the integration 'constants' $\phi^{ \pm}$(which are at most functions of $x_{1}$ and $\bar{z}$ ). The velocity jump corresponds to a logarithmic phase shift of $\pi$ when the critical layer is linear, which leads to the requirement that

$$
\phi^{+}-\phi^{-} \rightarrow \mathrm{i} \pi \quad \text { as } \quad x_{1} \rightarrow-\infty
$$

This ensures that the nonlinear solution (to be discussed below) will match onto the appropriate linear solution in the upstream region.

\subsection{The main boundary layer}

Equations (5.7) and (5.8) together with the expression for $\hat{p}_{y}$ obtained by substituting $Y=y / \sigma$ into (5.9) and re-expanding the result suggest that, in the main part of the boundary layer, $\hat{p}$ should expand like

$$
\hat{p}=\sigma\left(b_{0}+\sigma \hat{p}_{2}+\cdots\right)+\mathrm{i} \sigma^{4}\left(b_{3 i}+\sigma \hat{p}_{5 i}+\cdots\right)
$$

where $\hat{p}_{2}$ and $\hat{p}_{5 i}$ are real functions of $x_{1}, y$ and $\bar{z}$ and only the first two terms in the small- $\sigma$ expansions of the real and imaginary parts of $\hat{p}$ are shown. Substituting (3.11) into (3.7), inserting the result together with (5.17) into (4.15), and integrating with respect to $y$ yields

$$
\hat{p}_{2 y}=d_{2} f^{\prime 2}, \quad \hat{p}_{5 i y}=d_{5 i} f^{\prime 2}
$$

where $d_{2}$ and $d_{5 i}$ are at most functions of $x_{1}$ and $\bar{z}$. Matching with the real and imaginary parts of the wall-layer solution (5.9) then shows that

$$
d_{2}=\mathcal{D}_{0}\left(\frac{1}{\lambda_{0} \bar{c}_{0}}\right)
$$




$$
d_{5 i}=\mathcal{D}_{3 i}\left(\frac{1}{\lambda_{0} \bar{c}_{0}}\right)+\operatorname{Im}\left\{\mathcal{D}_{0}\left[\frac{\bar{\mu}}{\lambda_{0}^{3}}\left(\phi^{+}-\phi^{-}\right)-\frac{\mathrm{i} A^{t^{\prime}}}{\lambda_{0} \bar{S} A^{\dagger}}+\frac{1}{\sigma^{5} R^{\dagger} \bar{c}_{0}^{2} \bar{\omega}}\right]\right\}
$$

where

$$
\mathcal{D}_{0}=b_{0_{\bar{z}}} \partial / \partial \bar{z}+b_{0_{\bar{z} \bar{z}}}-\bar{\alpha}_{0}^{2} b_{0}
$$

and

$$
\mathcal{D}_{3 i}=b_{3 i \bar{z}} \partial / \partial \bar{z}+b_{3 i_{\bar{z} \bar{z}}}-\bar{\alpha}_{0}^{2} b_{3 i}+2 \bar{\alpha}_{0} \operatorname{Im}\left(\mathrm{i} A^{\dagger^{\prime}} / A^{\dagger}\right) b_{0}
$$

are the scaled leading-order approximations to the real and imaginary parts of $\mathcal{D}$.

\subsection{The outer layer}

Equation (5.18) shows that the solution (5.17) does not satisfy the free-stream boundary condition (4.20) and it is, therefore, necessary to construct the solution for $\hat{p}$ in the outer region described by (3.21). Substituting (3.21) into (5.17) and making use of (3.12) and (5.18) shows that, in this region, $\hat{p}$ expands like

$$
\hat{p}=\sigma \bar{p}_{1}+\cdots+\mathrm{i} \sigma^{4} \bar{p}_{4 i}+\cdots
$$

where $\bar{p}_{1}$ and $\bar{p}_{4 i}$ are real functions of $x_{1}, \bar{y}$ and $\bar{z}$ and only the leading-order terms in the small- $\sigma$ expansions of the real and imaginary parts of $\hat{p}$ are shown. $\bar{p}_{1}$ and $\bar{p}_{4 i}$ must satisfy

$$
\bar{p}_{1}=b_{0}, \quad \bar{p}_{1 \bar{y}}=d_{2}, \quad \bar{p}_{4 i}=b_{3 i}, \quad \bar{p}_{4 i_{\bar{y}}}=d_{5 i} \quad \text { at } \quad \bar{y}=0
$$

in order to match with the main-boundary-layer solution (5.17) and (5.18) and

$$
\bar{p}_{1}, \bar{p}_{4 i} \rightarrow 0 \quad \text { as } \quad \bar{y} \rightarrow \infty
$$

in order to satisfy the free-stream boundary condition (4.20). Equation (3.22) shows that the mean streamwise velocity $U$ approaches unity at a fast enough rate to ensure that (4.15) 
reduces to the Helmholtz equation when $\bar{y}=O(1)$. Therefore, substituting (4.16) and (5.23) into (4.15) leads to

$$
\begin{aligned}
& \bar{p}_{1_{\bar{y} \bar{y}}}+\bar{p}_{1_{\bar{z} \bar{z}}}-\bar{\alpha}_{0}^{2} \bar{p}_{1}=0 \\
& \bar{p}_{4 i_{\bar{y} \bar{y}}}+\bar{p}_{4 i_{\bar{z} \bar{z}}}-\bar{\alpha}_{0}^{2} \bar{p}_{4 i}=-2 \bar{\alpha}_{0} \operatorname{Im}\left(\mathrm{i} A^{t^{\prime}} / A^{\dagger}\right) \bar{p}_{1} .
\end{aligned}
$$

Up to this point, no restrictions have been placed on the $\bar{z}$ dependence of the solution. However, as indicated above, our interest is in the case where the upstream linear solution has a standing wave behavior in the spanwise direction with a spanwise wavelength twice that of the imposed cross-flow velocity $W_{0}$. This means that $b$ should be of the form

$$
b=2 \bar{\alpha} \cos \bar{\beta} \bar{z}+O\left(\sigma^{3}\right)
$$

where the normalization $2 \bar{\alpha}$ has been inserted for convenience.

It now follows from (5.19) and (5.24)-(5.26) that the relevant solution to (5.26) is

$$
\bar{p}_{1}=2 \bar{\alpha}_{0} \mathrm{e}^{-\bar{k} \bar{y}} \cos \bar{\beta} \bar{z}
$$

where

$$
\bar{k}=\left(\bar{\alpha}_{0}^{2}+\bar{\beta}^{2}\right)^{\frac{1}{2}}=\lambda_{0} \bar{c}_{0}
$$

The boundary-value problem (5.24), (5.25) and (5.27) only possesses solutions for certain values of $\operatorname{Im}\left(\mathrm{i} A^{\dagger^{\prime}} / A^{\dagger}\right)$ since $\bar{p}_{1}$ is a homogeneous solution to (5.27). These values can be found without explicitly solving for $\bar{p}_{4 i}$ by integrating the difference between $\bar{p}_{1}$ times $(5.27)$ and $\bar{p}_{4 i}$ times $(5.26)$ from $\bar{y}=0$ to $\infty$, integrating the result from $\bar{z}=0$ to $2 \pi / \bar{\beta}$ and then using (5.24), (5.25) and the $\bar{z}$-periodicity of $\hat{p}$ and $U$ to arrive at the following solvability condition

$$
\int_{0}^{2 \pi / \bar{\beta}}\left(b_{0} d_{5 i}-d_{2} b_{3 i}\right) \mathrm{d} \bar{z}=2 \bar{\alpha}_{0} \operatorname{Im}\left(\mathrm{i} A^{\dagger^{\prime}} / A^{\dagger}\right) \int_{0}^{2 \pi / \bar{\beta}} \int_{0}^{\infty} \bar{p}_{1}^{2} \mathrm{~d} \bar{y} \mathrm{~d} \bar{z}
$$


It now follows from (5.19)-(5.22), (5.29) and (5.30) that

$$
\begin{aligned}
& \left(\cos \theta+\frac{1}{\cos \theta}\right)\left(A^{\dagger^{\prime}}-\mathrm{i} \kappa_{i} A^{\dagger}\right)= \\
& -\mathrm{i} \frac{\bar{\beta}}{2 \pi} \int_{0}^{2 \pi / \bar{\beta}} \frac{\bar{c}_{0}^{2}}{\lambda_{0}} \bar{\mu}\left(\phi^{+}-\phi^{-}\right)(1+\cos 2 \theta \cos 2 \bar{\beta} \bar{z}) \mathrm{d} \bar{z} A^{\dagger}+\frac{\lambda_{0}^{2}}{\sigma^{5} R^{\dagger}(2 \bar{S})^{\frac{1}{2}}} A^{\dagger}
\end{aligned}
$$

where $\theta \equiv \arctan \left(\bar{\beta} / \bar{\alpha}_{0}\right)$ is the obliqueness angle of the instability wave and the real constant $\kappa_{i}$ has been introduced to account for the $O\left(\sigma^{3}\right)$ term in the expansion of the streamwise wavenumber in the upstream linear region. $\kappa_{i}$ is completely determined by the linear problem outside the critical layer but its explicit form is not given here because it is not needed for the subsequent analysis.

Equations (3.44), (3.45), (5.12) (5.14), (5.16) and (5.32) imply that

$$
A^{\dagger} \rightarrow A_{0}^{\dagger} \mathrm{e}^{\kappa x_{1}} \quad \text { as } \quad x_{1} \rightarrow-\infty
$$

where $A_{0}^{\dagger}$ is a constant, $\kappa=\kappa_{\tau}+\mathrm{i} \kappa_{i}$,

$$
\kappa_{r}=\frac{\cos \theta}{\cos ^{2} \theta+1}\left[\frac{\pi \bar{c}_{0}^{2}}{\lambda_{0}} \tilde{\Omega} \cos 2 \theta-\frac{\pi \bar{c}_{0}^{4}}{4 \lambda_{0}}+\frac{\lambda_{0}^{2}}{\sigma^{5} R^{\frac{1}{4}}(2 \bar{S})^{\frac{1}{2}}}\right]
$$

is the common initial parametric growth rate of the oblique modes and

$$
\tilde{\Omega} \equiv \frac{\bar{\beta}}{2 \pi} \int_{0}^{2 \pi / \bar{\beta}} \tilde{U}_{Y Y}\left(\ell, Y_{c}, \bar{z} ; \sigma\right) \cos (2 \bar{\beta} \bar{z}) \mathrm{d} \bar{z}
$$

with $\tilde{U}$ given by (3.44), (3.50) and (3.52). The last term in (5.34) accounts for the viscousStokes-layer effect and is negligible when $\sigma \gg R^{-\frac{1}{20}}$. The first term in (5.34) represents the growth produced by the resonant interaction with the streamwise vortices. It is always possible to make $\kappa_{r}$ positive by appropriately selecting the imposed cross-flow velocity $W_{0}$ which is now assumed to be the case. 


\section{Nonlinear-critical-layer effects}

Since (5.33) and (5.34) imply that the linear instability wave continues to grow as it propagates downstream, nonlinear effects eventually come into play and, as already noted, this first occurs locally within the so-called critical layer. The thickness of the critical layer (on the $y$ scale) turns out to be of the same order as the growth rate so the appropriate scaled transverse coordinate for this region is

$$
\bar{\eta}=\left(y-\sigma \bar{c} / \lambda_{0}\right) / \sigma^{4}=\left(Y-\bar{c} / \lambda_{0}\right) / \sigma^{3}
$$

Nonlinear terms produce a critical-layer velocity jump at the same order as the linear/ parametric-growth terms when the amplitude scale $\epsilon$, which was defined in (4.2), is chosen to be

$$
\epsilon=\sigma^{10}
$$

Viscous effects will enter into the dominant balance for the critical layer while making only insignificant modifications to the external flow when the Benney-Bergeron parameter

$$
\lambda \equiv 1 / \sigma^{13} R^{\frac{1}{2}}
$$

(Benney \& Bergeron 1969) is order one. The implied wavelength-Reynolds-number scaling (6.3) is more restrictive than (4.10) in the sense that the Stokes-layer contribution to (5.34) is negligible when $\lambda=O(1)$. However, retaining this term in (5.34) while assuming $\lambda=O(1)$ in the critical-layer analysis leads to a kind of distinguished scaling from which the more viscous case (4.10) can be recovered as a limit (see below) and we therefore adopt (6.3) as the appropriate wavelength-Reynolds-number scaling for the present problem. 
Equations (5.10) and (6.1) imply that

$$
Y-Y_{c}=\sigma^{3}\left(\bar{\eta}-\bar{\eta}_{c}\right)+\cdots
$$

where

$$
\bar{\eta}_{c}(\bar{z} ; \sigma) \equiv-\lambda_{0}^{-1} \tilde{U}\left(\ell, Y_{c}, \bar{z} ; \sigma\right)+\frac{1}{48} \lambda_{0} Y_{c}^{4}+\frac{1}{2} \ell Y_{c}
$$

accounts for spanwise variations in the position of the critical level. Introducing (6.4) into the expressions for $\hat{u}, \hat{v}, \hat{w}$ and $\hat{p}$ obtained from (4.17)-(4.19), (5.7) and (5.9), re-expanding the result, and using (3.33)-(3.35), (4.2), (4.3), (4.11), (6.2) and (6.3) shows that the criticallayer flow should expand like

$$
\begin{aligned}
& u=\sigma \bar{c}+\sigma^{4} \lambda_{0}\left(\bar{\eta}-\bar{\eta}_{c}\right)+\sigma^{7} u_{1}+\sigma^{10} u_{2}+\sigma^{13} u_{3}+\cdots \\
& v=\sigma^{12} v_{1}+\sigma^{15} v_{2}+\sigma^{18} v_{3}+\cdots \\
& w=\sigma^{7} w_{1}+\sigma^{10} w_{2}+\sigma^{13} w_{3}+\cdots \\
& p=\sigma^{-3} R^{-1} P_{D}+\sigma^{11} 2 \bar{\alpha} \cos \bar{\beta} \bar{z} \operatorname{Re}\left(A^{\dagger} \mathrm{e}^{\mathrm{i} X}\right)+\sigma^{14} p_{2}+\sigma^{17} p_{3}+\cdots
\end{aligned}
$$

where the $u_{n}, v_{n}, w_{n}$, and $p_{n}$ are functions of $X, x_{1}, \bar{\eta}$ and $\bar{z}$ that have expansions in $\sigma$ containing terms up to but not including $O\left(\sigma^{3}\right)$. Matching (6.6)-(6.9) with the external linear solution requires that

$$
\begin{aligned}
u_{1} \rightarrow\left[\tilde{U}_{Y}\left(\ell, Y_{c}, \bar{z} ; \sigma\right)-\right. & \left.\frac{1}{12} \lambda_{0}^{2} Y_{c}^{3}-\frac{1}{2} \lambda_{0} \ell\right] \bar{\eta} \\
& +2 \bar{\beta}^{2} \cos \bar{\beta} \bar{z} \operatorname{Re}\left(A^{\dagger} \mathrm{e}^{\mathrm{i} X}\right) / \lambda_{0} \bar{\alpha}\left(\bar{\eta}-\bar{\eta}_{c}\right)
\end{aligned}
$$

and

$$
w_{1} \rightarrow-2 \bar{\beta} \sin \bar{\beta} \bar{z} \operatorname{Re}\left(\mathrm{i} A^{\dagger} \mathrm{e}^{\mathrm{i} X}\right) / \lambda_{0}\left(\bar{\eta}-\bar{\eta}_{c}\right)
$$


as $\bar{\eta} \rightarrow \pm \infty$. Matching the critical layer induced velocity jump with the external jump (5.15) requires

$$
\frac{1}{\pi} \int_{-\infty}^{+\infty} \int_{0}^{2 \pi} \mathrm{e}^{-\mathrm{i} X} u_{3_{\bar{\eta}}} \mathrm{d} X \mathrm{~d} \bar{\eta}=-\mathcal{D}_{0}\left[\bar{\mu}\left(\phi^{+}-\phi^{-}\right) / \lambda_{0}^{2} \bar{\alpha}_{0}^{2}\right] A^{\dagger}
$$

Multiplying (6.12) by $\cos \bar{\beta} \bar{z}$, integrating from $\bar{z}=0$ to $2 \pi / \bar{\beta}$ and then combining the result with (5.32) yields the jump condition

$$
\begin{aligned}
\frac{\bar{\beta}}{2 \pi^{2}} \int_{0}^{2 \pi / \bar{\beta}} \int_{-\infty}^{+\infty} \int_{0}^{2 \pi} \mathrm{e}^{-\mathrm{i} X} u_{3 \bar{\eta}} \cos \bar{\beta} \bar{z} \mathrm{~d} X \mathrm{~d} \bar{\eta} \mathrm{d} \bar{z}= \\
\mathrm{i} \frac{\lambda_{0}}{\bar{\alpha}_{0}}\left[\left(\cos \theta+\frac{1}{\cos \theta}\right)\left(A^{\dagger^{\prime}}-\mathrm{i} \kappa_{i} A^{\dagger}\right)-\lambda_{0}^{2}\left(\frac{\sigma^{3} \lambda}{2 \bar{S}}\right)^{\frac{1}{2}} A^{\dagger}\right] .
\end{aligned}
$$

Notice that, for the reasons given above, the asymptotically small Stokes-layer term has been retained in this result.

Substituting the expansions (6.6)-(6.9) into (2.3) and (2.4) and combining the result with $(6.10),(6.11)$ and $(6.13)$ leads to a boundary-value problem for the $u_{n}, v_{n}, w_{n}$, and $p_{n}$. When this is expressed in terms of the strained coordinate

$$
\tilde{\eta}=\bar{\eta}-\bar{\eta}_{c}
$$

and the corresponding velocity component

$$
\tilde{v}_{n}=v_{n}-\bar{\eta}_{c_{\bar{z}}} w_{n}, \quad \text { for } \quad n=1,2,3
$$

we find that

$$
\tilde{v}_{1}=-2\left(\bar{\alpha}^{2}+\bar{\beta}^{2}\right) \cos \bar{\beta} \bar{z} \operatorname{Re}\left(\mathrm{i} A^{\dagger} \mathrm{e}^{\mathrm{i} X}\right) / \lambda_{0}
$$

and, more generally, that the entire problem is just the viscous version of the one solved in Goldstein \& Choi (1989), a special case of the one considered in Goldstein \& Lee (1992), and, except for differences in notation, precisely the one considered in Wu, Lee \& Cowley (1993). 
The spanwise variation of the mean flow (which is the new feature in the present analysis) now enters the problem only through the transverse boundary condition (6.10) as well as the transverse boundary conditions for $u_{2}, u_{3}, \tilde{v}_{2} \tilde{v}_{3}$ and $w_{3}$. But, since the spanwise variation of the mean flow introduced by these conditions only affects the linear/parametric-growth terms, the solution to the present critical-layer problem can be easily deduced from the solutions given in the previous investigations. Substituting this solution into the velocityjump condition (6.13) leads to the following equation for $A^{\dagger}$

$$
A^{\dagger^{\prime}}=\kappa A^{\dagger}+\mathrm{i} M \int_{-\infty}^{x_{1}} \int_{-\infty}^{\xi_{1}} K\left(x_{1} \mid \xi_{1}, \xi_{2}\right) A^{\dagger}\left(\xi_{1}\right) A^{\dagger}\left(\xi_{2}\right) A^{\dagger^{*}}\left(\xi_{1}+\xi_{2}-x_{1}\right) \mathrm{d} \xi_{1} \mathrm{~d} \xi_{2}
$$

where

$$
M \equiv 2 \pi \lambda_{0}^{4} \bar{\alpha}_{0}^{4} \cos ^{2} \theta /\left(\cos ^{2} \theta+1\right)
$$

the asterisk denotes complex conjugation and the initial parametric growth rate $\kappa_{r}$ is given by (5.34). The kernel function $K$ is fairly complicated in the general viscous (i.e. order-one ג) case considered by $\mathrm{Wu}$, Lee \& Cowley (1993) and Leib \& Lee (1994), but, in the inviscid limit first considered by Goldstein \& Choi (1989), it is a relatively simple polynomial of the streamwise coordinate and is given by

$$
K=\frac{1}{2} \tan ^{2} \theta \cos 2 \theta\left(x_{1}-\xi_{1}\right)\left[\cos 2 \theta\left(x_{1}-\xi_{1}\right)\left(\xi_{1}-\xi_{2}\right)-\left(x_{1}-\xi_{1}\right)^{2}-\left(x_{1}-\xi_{2}\right)^{2}\right] .
$$

As already indicated the Stokes-layer contribution to $\kappa_{\tau}$ is negligible when $\lambda=O(1)$. However, retaining this contribution and taking the limit as $\lambda / \kappa_{r}^{3} \rightarrow \infty$ of the integral term in (6.17) as was done by $\mathrm{Wu}$, Lee \& Cowley (1993), leads to the amplitude-evolution equation corresponding to the upper-branch Blasius-boundary-layer scaling $\sigma R^{\frac{1}{20}}=O(1)$. In the 
present notation, this equation is

$$
A^{\dagger^{\prime}}=\kappa A^{\dagger}-\mathrm{i} \bar{\lambda}^{-\frac{1}{3}} M \tan ^{2} \theta \sin ^{2} \theta \cos 2 \theta\left(\frac{1}{18}\right)^{\frac{1}{3}} \Gamma\left(\frac{1}{3}\right) A^{\dagger} \int_{-\infty}^{x_{1}}\left|A^{\dagger}(\xi)\right|^{2} \mathrm{~d} \xi,
$$

where $\bar{\lambda} \equiv \lambda_{0}^{2} \bar{\alpha}_{0}^{2} \lambda / \bar{c}_{0}^{3}$ is a rescaled Benney-Bergeron parameter.

\section{Results and discussion}

In one sense, the oblique-mode amplitude equation (6.17) (or its highly viscous limit $(6.20))$ can be thought of as the final result of this paper. While this equation is now well known, its application to the present situation is new as is the formula (5.34) for the initial parametric growth rate of the oblique modes $\kappa_{r}$. The latter accounts for the parametricresonance effects that allow the oblique modes to grow faster than the two-dimensional waves which, in turn, allows the oblique-mode interaction described by (6.17) to become the dominant interaction in the initial nonlinear stage of the transition process. The initial parametric-resonant interaction takes place between the relatively weak spanwise-periodic mean-flow distortion (i.e. the streamwise vortices) and a pair of equal-amplitude oblique modes that form a standing wave in the spanwise direction with spanwise wavelength equal to twice that of the mean-flow distortion. Equation (5.34) shows that its spatial growth rate is enhanced by the inflectional nature of the mean velocity profile.

The transverse position of the mean-flow inflection point $y_{s}=\sigma Y_{s}$ is determined by

$$
\tilde{U}_{Y Y}-\frac{1}{4} \lambda_{0}^{2} Y^{2}=0 \text { at } Y=Y_{s}
$$

Figure 1 shows how $Y_{s}$ varies with $x_{2}$ for an initial spanwise velocity that behaves like

$$
B(\bar{z}) \equiv W_{0}(0, \bar{z})=(2 \bar{\beta})^{-1} B_{0} \sin 2 \bar{\beta} \bar{z}
$$




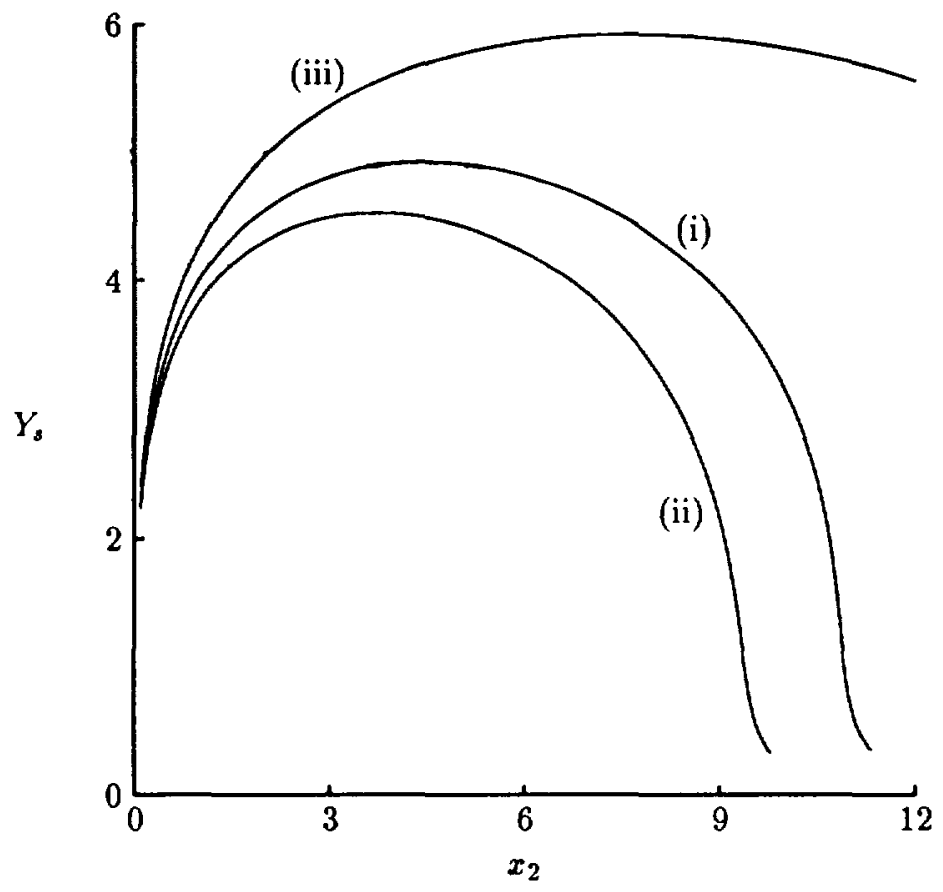

Figure 1: Transverse position of mean-flow inflection point on the $\bar{z}=0$ plane vs. downstream distance for $\sigma=0.05$. (i) $B_{0}=-12 \pi, \bar{\beta}=\pi / 24$; (ii) $B_{0}=-9 \pi, \bar{\beta}=\pi / 24$; (iii) $B_{0}=-16 \pi, \bar{\beta}=\pi / 18$. 
at the wall. For this class of imposed cross flows, (3.20), (3.29) and (3.51) can be solved analytically to obtain

$$
\mathcal{A}=\sum_{m=4}^{6}(-\bar{\chi})^{\frac{m}{3}-1} \int_{0}^{x_{2}} Q_{\bar{z}}^{(m)}(\xi, \bar{z} ; \sigma) \mathrm{e}^{-\bar{\chi}\left(x_{2}-\xi\right)} \mathrm{d} \xi
$$

and

$$
P_{D_{\bar{z} \bar{z}}}=2 \bar{\beta} \mathcal{A}_{x_{2} x_{2}}
$$

where $\bar{\chi} \equiv 9 \lambda_{0}^{5} /\left[2 \bar{\beta} \Gamma\left(\frac{1}{3}\right)\right]^{3}$. The $O(1 / \ln \sigma)$ terms in (3.44) and (3.53) were kept when computing the results presented here since these terms can be significant even at relatively small values of $\sigma$. The mean-flow distortion $\tilde{U}$ initially grows linearly with increasing $x_{2}$ (see (3.7), (3.15), (3.33), (3.44), (3.52) and (3.55)) causing $Y_{s}$ to move out from the wall. This type of linear disturbance growth is similar to the algebraic growth proposed by Ellingsen \& Palm (1975), Hultgren \& Gustavsson (1981) and Landahl (1990) as an alternative or 'bypass' transition mechanism. However, only the mean-flow distortion (or vortex flow) undergoes this type of growth in the present study and the follow-on 'secondary' instability modes exhibit the more conventional exponential-type growth. In the present analysis, the initial mean-flow growth is eventually reversed by viscous effects once the viscous wall layer expands to fill the entire Blasius boundary layer, i.e. once $x_{2}$ becomes $O\left(\sigma^{-3}\right)$. However, figures 1 and 2 show that the mean-flow inflection point produced by the distortion actually vanishes before this stage is reached.

The corresponding initial parametric growth rate $\kappa_{r}$ is shown in figure 3 . Near the excitation device, i.e. at small values of $\ell$, the mean-flow inflection point lies very close to the wall and, as shown by the figure, $\kappa_{r}$ is negative when $\sigma R^{\frac{1}{26}}=O(1)$ and given by the TollmienSchlichting-wave result when $\sigma R^{\frac{1}{20}}=O(1)$. The actual unsteady flow in this region would 


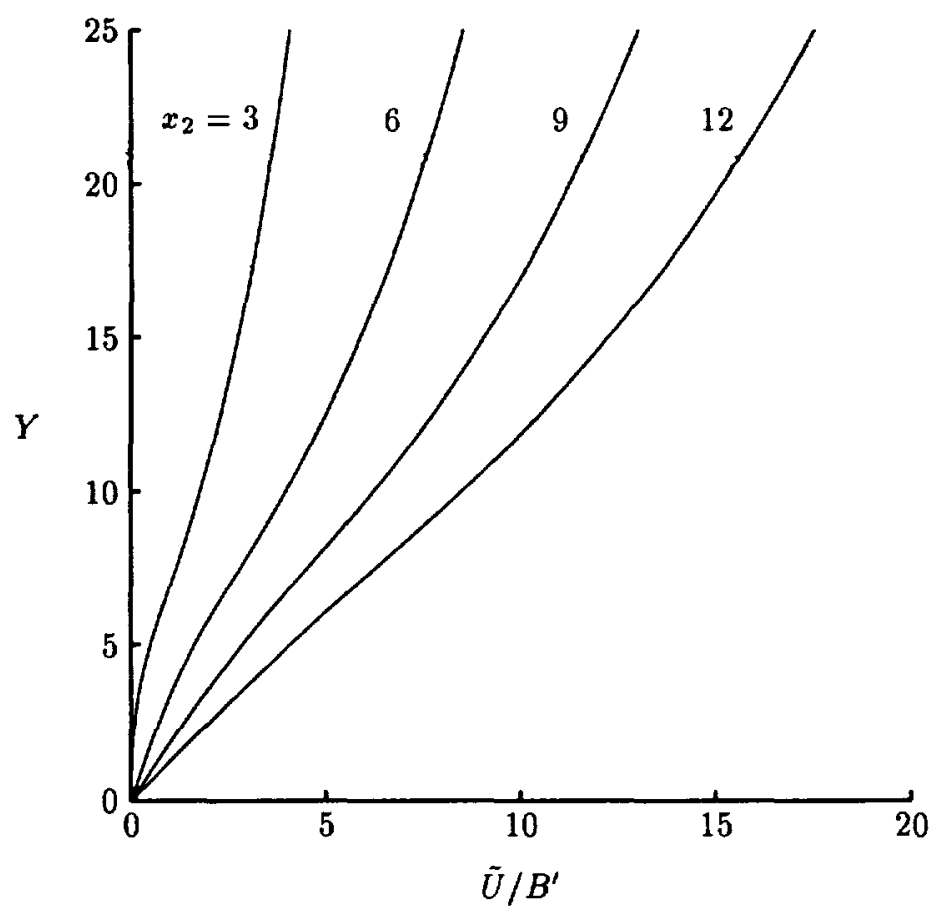

Figure 2: Scaled streamwise velocity profiles of the vortex flow within the viscous wall layer for $\sigma=0.05, \bar{\beta}=\pi / 24$ and various values of $x_{2}$. 

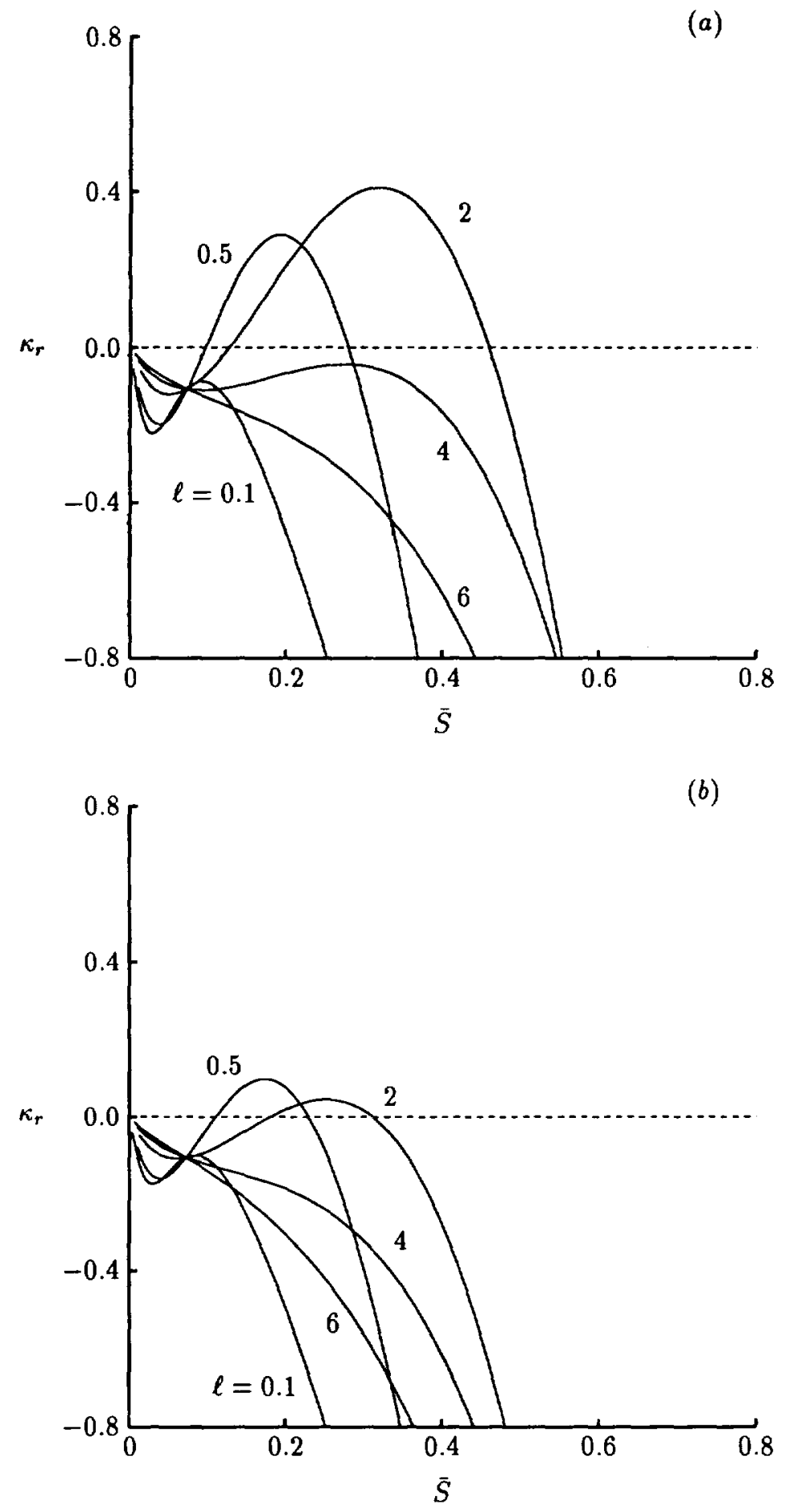

Figure 3: Initial parametric growth rate vs. scaled Strouhal number for $\sigma=0.05$ and various values of $\ell$. (a) $B_{0}=-12 \pi, \bar{\beta}=\pi / 24, \lambda=0$; (b) $B_{0}=-9 \pi, \bar{\beta}=\pi / 24, \lambda=0$; (c) $B_{0}=-16 \pi, \bar{\beta}=\pi / 18, \lambda=0$; (d) $B_{0}=-12 \pi, \bar{\beta}=\pi / 24, \lambda=1 / \sigma^{3}$. 

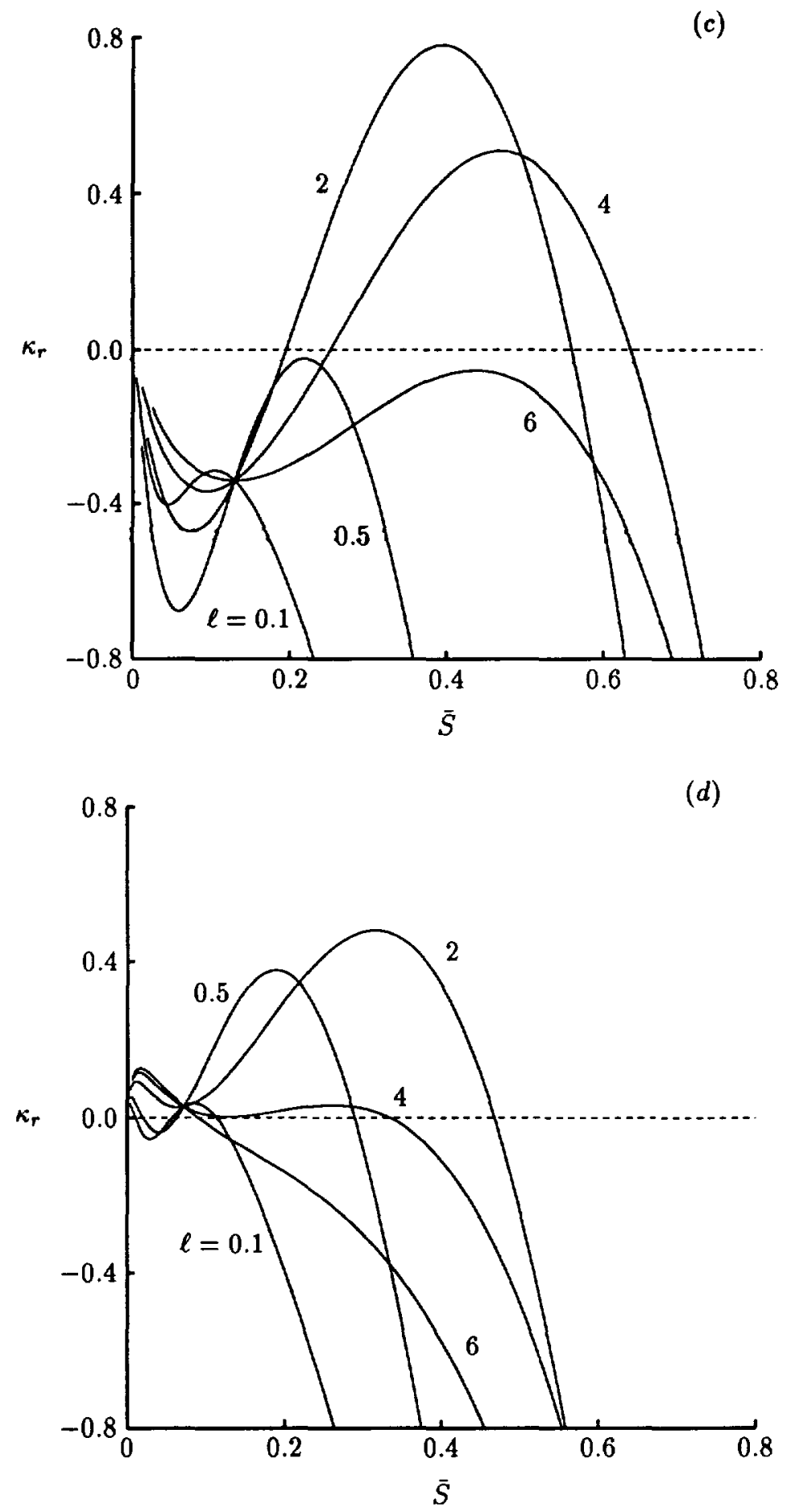

Figure 3: Initial parametric growth rate vs. scaled Strouhal number for $\sigma=0.05$ and various values of $\ell$. (a) $B_{0}=-12 \pi, \bar{\beta}=\pi / 24, \lambda=0$; (b) $B_{0}=-9 \pi, \bar{\beta}=\pi / 24, \lambda=0$; (c) $B_{0}=-16 \pi, \bar{\beta}=\pi / 18, \lambda=0$; (d) $B_{0}=-12 \pi, \bar{\beta}=\pi / 24, \lambda=1 / \sigma^{3}$. 
probably be dominated by two-dimensional viscous instabilities, i.e. by Tollmien-Schlichting waves, with the three-dimensional inviscid instabilities emerging further downstream where the mean-flow distortion has become sufficiently large. The continued growth of this distortion can then lead to much larger growth rates for the three-dimensional instabilities than those of the Tollmien-Schlichting waves. This is consistent with the experimental observations, which probably correspond to the case where $\sigma R^{\frac{1}{20}}=O(1)$, and show that the three-dimensional structures (which can be identified with the oblique modes described by the present analysis) gradually emerge from the initial two-dimensional motion. Figures 3 and 4 suggest that these structures will eventually decay once the mean-flow inflection point has been eliminated by viscous effects. However, the oblique modes will most likely become nonlinear before this occurs and their amplitudes will then determined by (6.17).

Since the nonlinear critical-layer equations (when expressed in terms of $\bar{\eta}$ ) explicitly involve the spanwise variation of the critical-level position, it would have been impossible to anticipate that the nonlinear oblique-mode amplitude would be determined by (6.17) which had previously been derived only for nonlinear interactions on strictly two-dimensional mean flows. However, the transformation (6.14) and (6.15) eliminates this spanwise variation from the problem and the two-dimensional mean-flow equation (6.17) is therefore obtained. This equation can be rescaled to eliminate the parameters $\kappa$ and $M$ (see Goldstein \& Choi 1989; Wu, Lee \& Cowley 1993; Leib \& Lee 1994) so that the resulting solutions depend only on the obliqueness angle $\theta$ and the scaled viscous/growth-rate parameter $\bar{\lambda} / \kappa_{r}^{3}$.

The numerical results for the rescaled oblique-mode amplitude are relatively universal and effectively the same as those given by Wu, Lee \& Cowley (1993) in the viscous case and by Goldstein \& Choi (1989) in the inviscid limit. We therefore do not present any new 

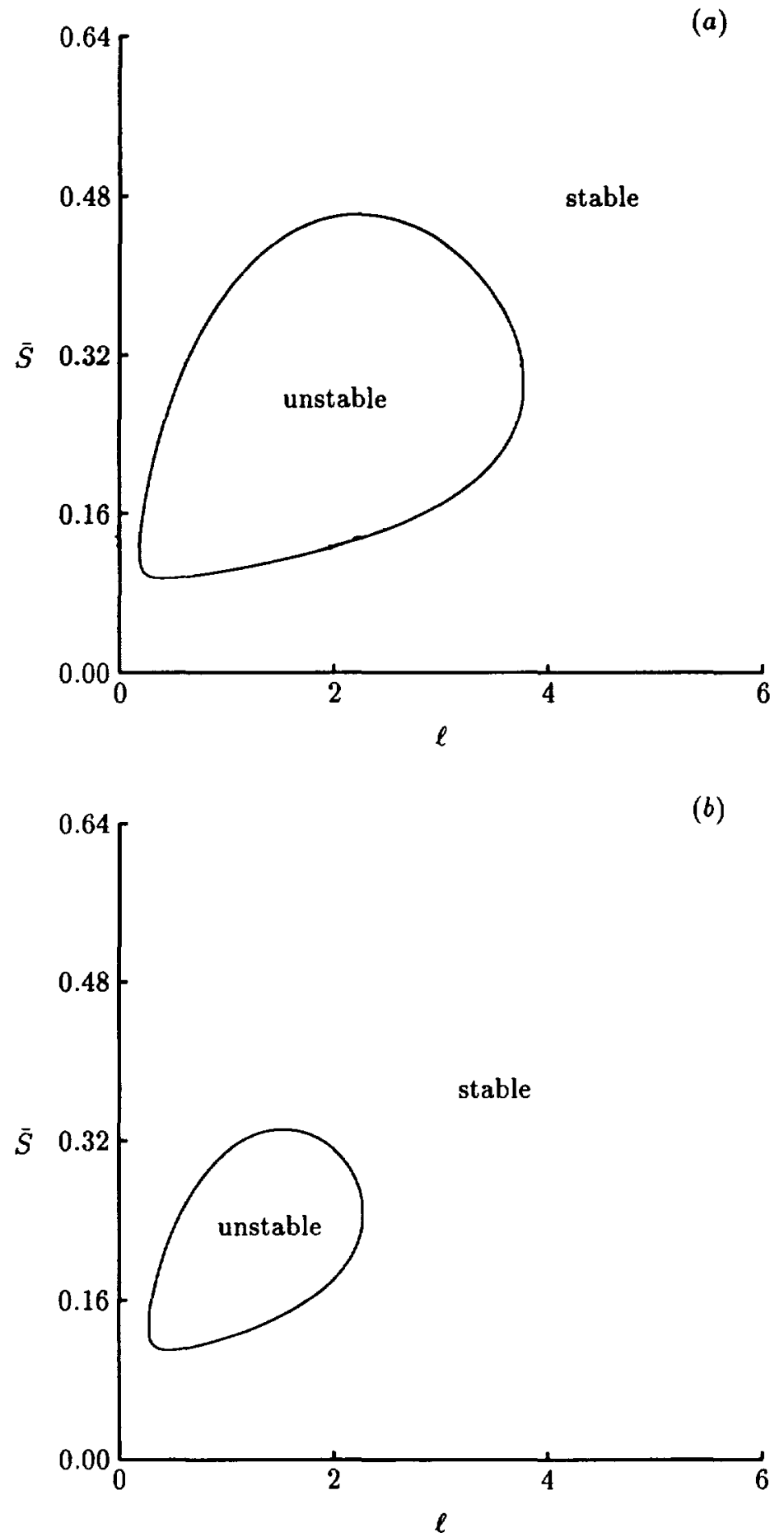

Figure 4: Scaled neutral Strouhal number vs. downstream distance for $\sigma=0.05$. (a) $B_{0}=-12 \pi, \bar{\beta}=\pi / 24, \lambda=0$; (b) $B_{0}=-9 \pi, \bar{\beta}=\pi / 24, \lambda=0$; (c) $B_{0}=-16 \pi, \bar{\beta}=\pi / 18$, $\lambda=0 ;$ (d) $B_{0}=-12 \pi, \bar{\beta}=\pi / 24, \lambda=1 / \sigma^{3}$. 

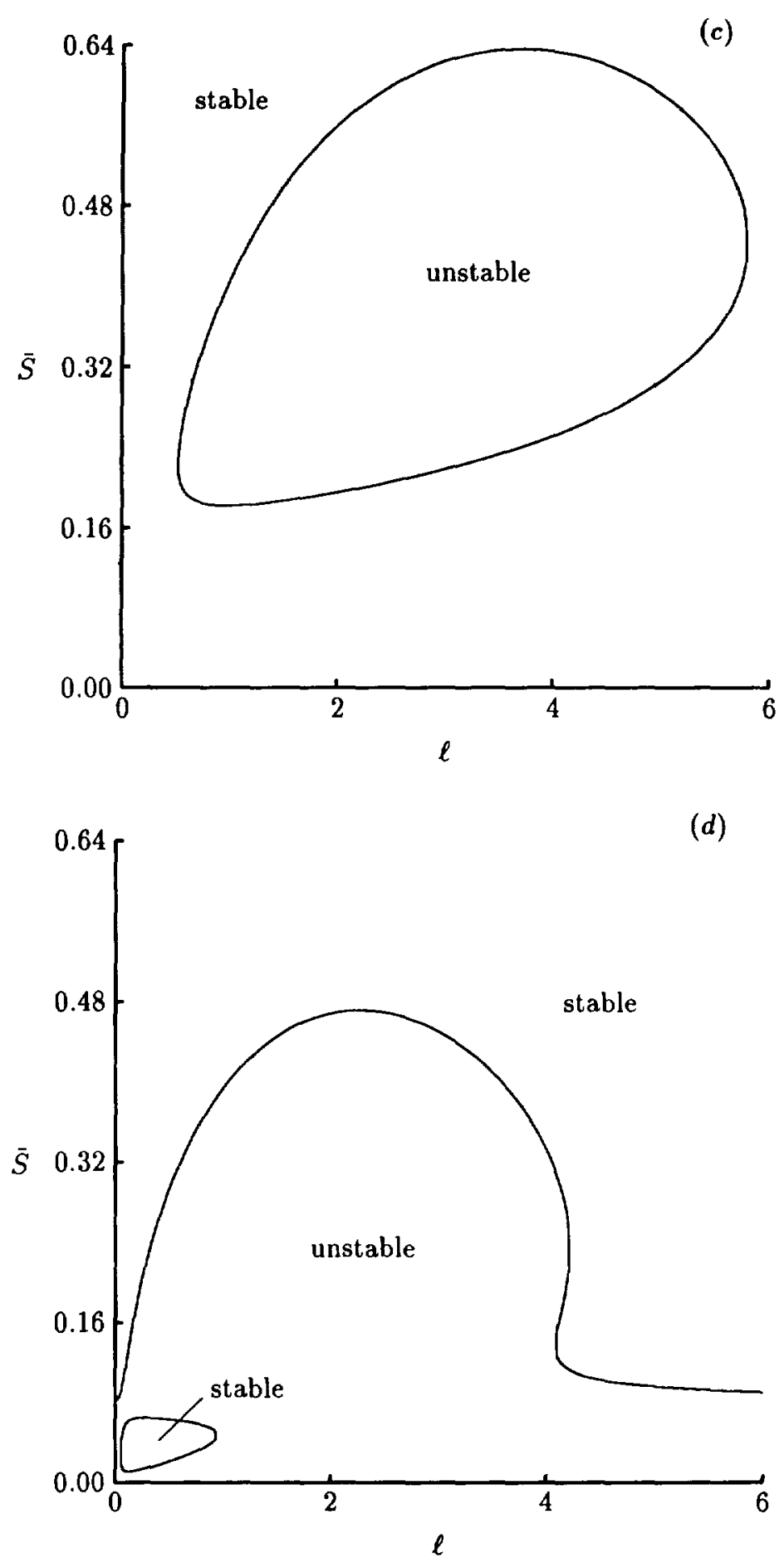

Figure 4: Scaled neutral Strouhal number vs. downstream distance for $\sigma=0.05$. (a) $B_{0}=-12 \pi, \bar{\beta}=\pi / 24, \lambda=0 ;$ (b) $B_{0}=-9 \pi, \bar{\beta}=\pi / 24, \lambda=0 ;$ (c) $B_{0}=-16 \pi, \bar{\beta}=\pi / 18$, $\lambda=0 ;$ (d) $B_{0}=-12 \pi, \bar{\beta}=\pi / 24, \lambda=1 / \sigma^{3}$. 
computations for $A^{\dagger}$, but merely note that, as was shown by Goldstein \& Choi (1989), the solutions to (6.17) become singular at some finite downstream position, say $x_{s}$, and that the local asymptotic expansion in the vicinity of this singularity is

$$
A^{\dagger} \sim a_{s}\left(x_{s}-x_{1}\right)^{-3-\mathrm{i} \varphi}
$$

where $a_{s}$ and $\varphi$ are constants that are determined explicitly in Goldstein \& Choi (1989). This suggests that the initially linear instability waves can exhibit nonlinear break down before the mean-flow distortion (which supports these waves) is able to decay. This is analogous to the argument used by Cowley (1987) and Wu, Lee \& Cowley (1993) to explain the breakdown of Stokes layers which (unlike the experimentally observed flows) would exhibit global stability over each oscillation cycle if nonlinear breakdown did not occur.

It is easy to solve the highly viscous amplitude equation (6.20) analytically and thereby show that the oblique modes continue to grow linearly in this limit. However, this solution shows that the wavenumber correction $\operatorname{Re}\left(\sigma^{4} A^{\dagger^{\prime}} / \mathrm{i} A^{\dagger}\right)$ increases exponentially with increasing $x_{1}$-indicating that the assumed wavenumber scaling must eventually break down. The next stage of evolution should be governed by the full non-equilibrium equation (6.17). The highly viscous solutions to this equation indicate that the explosive growth still occurs in this case (Dr. Sang Soo Lee, private communication) - which suggests that explosive growth will occur even when $\sigma R^{\frac{1}{20}}=O(1)$.

The amplitude $B^{\dagger}$ of the spanwise-variable mean-flow distortion $\operatorname{Re}\left[B^{\dagger}\left(x_{1}\right) \hat{g}(y, \bar{z})\right]$ induced on the external linear flow by the nonlinear critical-layer interactions is given by Goldstein (1994) as

$$
B^{\dagger}=b_{s} \int_{-\infty}^{x_{1}} \int_{-\infty}^{\xi_{1}}\left(x_{1}-\xi_{1}\right) \mathrm{e}^{-\frac{2}{3} \bar{\lambda}\left(\xi_{1}-\xi_{2}\right)^{3}}\left|A^{\dagger}\left(\xi_{2}\right)\right|^{2} \mathrm{~d} \xi_{2} \mathrm{~d} \xi_{1}
$$


which shows that $B^{\dagger}$ also becomes singular when $x_{1} \rightarrow x_{s}$ and behaves like

$$
B^{\dagger} \sim \frac{1}{60} b_{s}\left|a_{s}\right|^{2}\left(x_{s}-x_{1}\right)^{-3}
$$

in this limit.

Equations (3.7), (3.15) (3.19), (3.33), (4.2), (4.11) and (6.2) show that this induced spanwise-variable mean-flow velocity exceeds the initially imposed distortion velocity (in both the wall layer and main boundary layer) when

$$
x_{3}-x_{1}<\sigma^{2}
$$

The corresponding large amplitudes can occur without violating the present asymptotic scaling because the instability wave growth is self-induced and does not depend on the initial parametric growth once the oblique-mode amplitude becomes sufficiently large.

Of course, the oblique-mode amplitude and associated spanwise-variable mean-flow distortion cannot continue to increase indefinitely, and a new stage of evolution must eventually be reached when, as pointed out by Goldstein \& Lee (1992), the growth rate $\operatorname{Re}\left(\sigma^{4} A^{\dagger^{\prime}} / A^{\dagger}\right)$ becomes of the order of the wavelength scale $\sigma$, i.e. when

$$
x_{s}-x_{1}=O\left(\sigma^{3}\right)
$$

which is much shorter than the length scale (7.8) at which the induced mean-flow distortion exceeds the one imposed on the flow. The unsteady flow and spanwise distortions are now $O\left(\sigma U_{\infty}\right)$ in this smaller region and evolve on the relatively short wavelength scale $\bar{x}$. The resulting motion is, as noted in Goldstein \& Lee (1992), primarily inviscid and determined by the triple-deck equations (Stewartson 1969; Messiter 1970), but with no viscous terms appearing in the nonlinear lower-deck equations. This latter region has the same thickness 
as the wall layer associated with the original vortex system. There must, of course, be a thin viscous region underlying the triple-deck structure. The flow in this lower region, which is governed by the usual three-dimensional boundary-layer equations with the externally imposed pressure gradients determined by the flow in the triple deck, can, as pointed out by Smith \& Burggraf (1985), undergo large scale separation. However, it would not be possible to determine the flow in the triple deck, even if separation did not occur, because the downstream boundary conditions for this effectively elliptic problem depend on the downstream flow which is, in essence, turbulent in the situation of interest here.

While the relationship between the spanwise-wavelength scale $\sigma$ and the Reynolds number $R$ has not been completely fixed in the present analysis, we have required that

$$
1 \gg \sigma \gg 1 / R^{\mathbf{t}}
$$

The mean-flow distortion would be governed by the full three-dimensional triple-deck equations in the limit $\sigma \rightarrow 1 / R^{t}$. The present scaling corresponds to the short-wavelength limit of the triple-deck problem which was used by Rozhko \& Ruban (1987) and subsequently by Choudhari, Hall \& Streett (1992) to study Görtler vortices. However, the most appropriate distinguished scaling for the present problem corresponds to the order-one-wavelength limit $\sigma \rightarrow 1$. We chose to consider the long-wavelength limit $\sigma \ll 1$ in the hope that the analytical solutions that were obtainable in this limit would lead to a better understanding of the physical mechanisms involved.

Large scale numerical computations are required when $\sigma=1$ since the spanwisevariable mean flow (3.3) and (3.4) can only be determined by solving the parabolized Navier-Stokes equations (3.5) and (3.6). The relevant solutions still grow linearly with 
increasing downstream distance when $x_{2}$ is sufficiently small. In fact they are still given by (3.7)-(3.10), but with $\sigma$ and $\bar{\sigma}$ set equal to unity and with $U_{D}, V_{D}, W_{D}$ and $P_{D}$ given by (3.54), (3.17) and (3.55), respectively. This shows that the algebraic growth of the steady distortion is still important when $\sigma=1$.

While the initial linear instability wave can still be determined from the generalized Rayleigh equation (4.15) once the mean-flow velocity $U$ is known, the relevant solutions must now be found numerically since $\sigma=1$. However, the long-wavelength solutions of (4.15) show that there is an effective upper branch to the neutral stability curve (see figure 3 ) primarily because the mean-flow distortion ultimately decays to zero and thereby causes the inviscid instability wave growth rate (which is produced by this distortion) to vanish long before this decay is complete. Since this behavior also occurs when $\sigma=1$, all instability waves with sufficiently small initial amplitudes will now remain linear until their growth rates become sufficiently small. (Recall that the nonlinear amplitude of the instability waves scales with the growth rate and nonlinearity therefore occurs at smaller amplitudes when the growth rates become smaller.)

The nonlinearity will then occur within a thin critical layer with the solutions outside this layer still given by (4.11) and (4.15)-(4.20). Hall \& Horseman (1991), Horseman (1991) and Hall \& Smith (1991) have studied the local critical-level behavior of the solutions to the generalized Rayleigh equation and have shown that it is nearly identical to that of the usual three-dimensional solutions to the Rayleigh equation for strictly two-dimensional mean flows. In a sense, all solutions of the Rayleigh problem for spanwise-variable mean flows behave like three-dimensional solutions to the Rayleigh problem for strictly two-dimensional mean flows in the vicinity of their critical levels. This is most easily shown by expressing 
(4.15) in orthogonal curvilinear coordinates with one set of coordinate surfaces corresponding to surfaces of constant mean-flow velocity $U$ - as was done, for example, by Goldstein $(1976$, pp. 6-10).

The similarity of the solutions within the critical layer itself is even more dramatic. The critical-layer nonlinearity is still weak in the sense that it enters through an inhomogeneous term in a higher-order problem rather than through a coefficient in the lowest-order or dominant-balance equation and the instability wave amplitude $A^{\dagger}\left(x_{1}\right)$ can therefore still be determined from a single amplitude-evolution equation, which is again given by (6.17) but with the inviscid kernel function $K$ (which is still a simple polynomial of the streamwise coordinate) given by a slight generalization of the result (6.19).

Appendix A. Solutions for $\tilde{U}^{(I)}$ and $\tilde{W}^{(I)}$

In this appendix, solutions for $\tilde{U}^{(I)}$ and $\tilde{W}^{(I)}$ are constructed by first substituting (3.44) into (3.37) and (3.39)-(3.43) and then taking the Laplace transform of the resulting equations with respect to $x_{2}$ to obtain

$$
\begin{gathered}
\lambda_{0} s Y \check{W}^{(I)}+\check{P}_{D_{\bar{z}}}=\check{W}_{Y Y}^{(I)}, \\
\lambda_{0} s Y \check{U}_{Y}^{(I)}-\lambda_{0} \check{W}_{\bar{z}}^{(I)}=\check{U}_{Y Y Y}^{(I)}, \\
\check{W}^{(I)}=\check{U}^{(I)}=\check{U}_{Y Y}^{(I)}=0 \text { at } Y=0,
\end{gathered}
$$

and

$$
\check{W}^{(I)} \rightarrow 0, \quad \check{U}^{(I)} \rightarrow \lambda_{0}\left(\check{Q}_{\bar{z}}^{(6)}+\check{\mathcal{A}}\right) \quad \text { as } \quad Y \rightarrow \infty
$$

where

$$
(:) \equiv \int_{0}^{\infty} \mathrm{e}^{-s x_{2}}(\cdot) \mathrm{d} x_{2}
$$


denotes the Laplace transform with respect to $x_{2}$,

$$
\begin{gathered}
\check{Q}^{(m)}(s, \bar{z} ; \sigma) \equiv \bar{\sigma} \lambda_{0}^{-1} s^{-\frac{m}{3}}\left\{\ln \left[\sigma\left(\lambda_{0} s\right)^{-\frac{1}{3}}\right]-C_{1}+\frac{1}{3} \psi(2)\right\} B(\bar{z}), \\
C_{1} \equiv \lim _{\eta \rightarrow \infty}(F-\ln \eta)
\end{gathered}
$$

and $\psi$ denotes the digamma function defined on page 258 of Abramowitz \& Stegun (1964).

The relevant solutions to (A 1) and (A 2) are

$$
\check{W}^{(I)}=-\pi\left(\lambda_{0} s\right)^{-\frac{2}{3}} \dot{P}_{D_{\bar{z}}}\left[\mathrm{Gi}(\check{\eta})-3^{-\frac{1}{2}} \mathrm{Ai}(\check{\eta})\right]
$$

and

$$
\check{U}^{(I)}=\lambda_{0} \pi\left(\lambda_{0} s\right)^{-\frac{s}{3}} \check{P}_{D_{\bar{z} \bar{z}}}\left[\mathrm{Gi}(\check{\eta})-3^{-\frac{1}{2}} \mathrm{Ai}(\check{\eta})-3^{\frac{1}{3}} \Gamma\left(\frac{1}{3}\right) \frac{1}{\pi} \int_{0}^{\check{\eta}} \mathrm{Ai}(t) \mathrm{d} t\right]
$$

where $\check{\eta} \equiv\left(\lambda_{0} s\right)^{\frac{1}{3}} Y$ and $\mathrm{Ai}$ and $\mathrm{Gi}$ are the Airy functions defined on pages 446 and 448 of Abramowitz \& Stegun (1964). It follows from (A 4), (A 9) and the asymptotic behavior of the Airy functions for large values of their argument (Abramowitz \& Stegun 1964, pp. 448-450) that

$$
\left(9 \lambda_{0}^{5}\right)^{-\frac{1}{3}} \Gamma\left(\frac{1}{3}\right) \check{P}_{D_{\bar{z} \bar{z}}}=-\check{Q}_{\bar{z}}^{(1)}-s^{\frac{5}{3}} \check{\mathcal{A}}
$$

which can be inverted to give (3.51).

Before inverting (A 8) and (A 9), it is convenient to rewrite these equations as

$$
\check{W}^{(I)}=-\lambda_{0}^{-1} \Gamma\left(\frac{1}{3}\right)^{-1}\left(\lambda_{0} s\right)^{\frac{1}{3}} \check{P}_{D_{\bar{z}}} \check{G}^{(I)}(s, Y),
$$

and

$$
\check{U}^{(I)}=\Gamma\left(\frac{\mathbf{1}}{3}\right)^{-1}\left(\lambda_{0} s\right)^{-\frac{2}{3}} \dot{P}_{D_{\bar{z} \bar{z}}} \check{F}^{(I)}(s, Y),
$$

where $\check{G}^{(I)}$ and $\check{F}^{(I)}$ are the Laplace transforms with respect to $x_{2}$ of the functions of $\eta \equiv\left(\lambda_{0} / x_{2}\right)^{\frac{1}{3}} Y$ determined by

$$
G^{(I)^{\prime \prime}}+\frac{1}{3} \eta^{2} G^{(I)^{\prime}}=-1, \quad F^{(I)^{\prime \prime \prime}}+\frac{1}{3} \eta^{2} F^{(I)^{\prime \prime}}+\frac{1}{3} \eta F^{(I)^{\prime}}=-\frac{1}{3} \eta G^{(I)^{\prime}}
$$


with

$$
G^{(I)}=F^{(I)}=F^{(I) \prime}=0 \quad \text { at } \quad \eta=0
$$

and

$$
G^{(I)} \rightarrow 0, \quad F^{(I)} \rightarrow-3^{-\frac{2}{3}} \Gamma\left(\frac{1}{3}\right)^{2} \text { as } \eta \rightarrow \infty
$$

It follows from these equations that

$$
G^{(I)}=3^{-\frac{2}{3}} \Gamma\left(\frac{1}{3}\right) \gamma\left(\frac{1}{3}, \tau\right)+3^{-\frac{2}{3}} \int_{0}^{\tau} t^{-\frac{2}{3}} \mathrm{e}^{-t} \gamma\left(\frac{1}{3},-t\right) \mathrm{d} t
$$

and

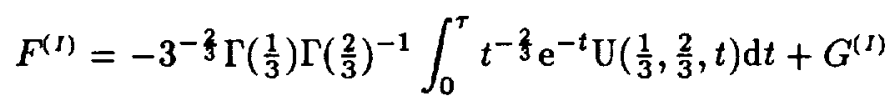

where $\tau \equiv \eta^{3} / 9$ and $U$ denotes the confluent hypergeometric function in the notation of Abramowitz \& Stegun (1964, p. 504). Using (A 10) when inverting (A 12) then leads to $(3.52)$.

Appendix B. Mean-flow solution for $x=O(\sigma)$

In this appendix, we briefly discuss the mean-flow solution in the streamwise region characterized by (3.56). The lowest-order main-deck velocity field is a simple re-expansion of the solution (3.15)-(3.17) but, as can be anticipated from (3.29) and (3.55), with the particle displacement $\mathcal{A}$ set equal to zero (i.e. it is now of higher order). Equation (3.55) also implies that the pressure in this region should expand like

$$
P=\bar{\sigma} \Delta \bar{\Delta}^{-1} R^{-\frac{1}{2}} \hat{P}_{D}+\cdots
$$

where $\Delta \equiv \sigma^{-\frac{1}{3}} R^{-\frac{1}{6}} \ll 1, \bar{\Delta} \equiv 1 / \ln \Delta$ and $\hat{P}_{D}$ is a function of $\hat{x}$ and $\bar{z}$ that has the implicit $\Delta$ dependence

$$
\hat{P}_{D}=\hat{P}_{0}(\hat{x}, \bar{z})+\bar{\Delta} \hat{P}_{1}(\hat{x}, \bar{z})
$$


Equations (3.7), (3.15) and (3.56) imply that the streamwise velocity distortion is now $O\left(R^{-\frac{1}{2}}\right)$, i.e. of the same order as the imposed cross-flow velocity that produced it.

The main difference from the $x=O\left(\sigma^{3} R^{\frac{1}{2}}\right)$ solution comes in the viscous wall layer which now corresponds to

$$
\hat{Y} \equiv y / \Delta=O(1)
$$

The velocity in this region expands like

$$
\begin{aligned}
U & =\Delta \lambda_{0} \hat{Y}+\bar{\sigma} \bar{\Delta}^{-1} R^{-\frac{1}{2}} \hat{U}+\cdots, \\
V & =\sigma \bar{\sigma} \Delta \bar{\Delta}^{-1} R^{-\frac{1}{2}} \hat{V}+\cdots \\
W & =\bar{\sigma} \bar{\Delta}^{-1} R^{-\frac{1}{2}} \hat{W}+\cdots
\end{aligned}
$$

where, like $\hat{P}_{D}$, the functions $\hat{U}, \hat{V}$, and $\hat{W}$ of $\hat{x}, \hat{Y}$, and $\bar{z}$ depend implicitly on $\Delta$. The flow in this region is determined by

$$
\begin{gathered}
\lambda_{0} \hat{Y} \hat{U}_{\hat{x}}+\lambda_{0} \hat{V}+\hat{P}_{D_{\hat{x}}}=\hat{U}_{\hat{Y} \hat{Y}} \\
\lambda_{0} \hat{Y} \hat{W}_{\hat{x}}+\hat{P}_{D_{\bar{z}}}=\hat{W}_{\hat{Y} \hat{Y}} \\
\hat{U}_{\hat{x}}+\hat{V}_{\hat{Y}}+\hat{W}_{\bar{z}}=0
\end{gathered}
$$

with

$$
\begin{gathered}
\hat{U}=\hat{V}=\hat{W}=0 \quad \text { at } \quad \hat{Y}=0, \\
\hat{W} \rightarrow \bar{\Delta} B, \quad \hat{U} \rightarrow \bar{\Delta} \hat{x} B^{\prime} \ln (\Delta \hat{Y}) \quad \text { as } \quad \hat{Y} \rightarrow \infty,
\end{gathered}
$$

and

$$
\hat{U} \rightarrow 0, \quad \hat{W} \rightarrow \bar{\Delta} B \quad \text { as } \quad \hat{x} \rightarrow 0 .
$$


The only difference from (3.36)-(3.41) is that the pressure gradient now appears in the streamwise momentum equation (B 7) and the particle displacement $\mathcal{A}$ does not appear in the boundary condition (B 11). This means that the pressure is completely determined by the solution in the lower deck and the now higher-order particle displacement is determined after the fact from the upper-deck problem. The solution procedure is essentially the same as before. Since the similarity variable $\eta$ remains order one when expressed in terms $\hat{x}$ and $\hat{Y}$, it is again appropriate to seek a solution of the form

$$
\hat{U}=\bar{\Delta} \hat{x} B^{\prime}(\bar{z}) F(\eta)+\hat{U}^{(I)}(\hat{x}, \hat{Y}, \bar{z} ; \Delta), \quad \hat{W}=\bar{\Delta} B(\bar{z}) G(\eta)+\hat{W}^{(I)}(\hat{x}, \hat{Y}, \bar{z} ; \Delta)
$$

where the Laplace transforms of $\hat{W}^{(I)}$ and $\hat{U}^{(I)}$ are determined by (A 1) and (A 2) but with $Y$ replaced by $\hat{Y}$ and with the boundary conditions now given by

$$
\check{W}^{(I)}=\check{U}^{(I)}=0, \quad \check{U}_{\tilde{Y} \grave{Y}}^{(I)}=s \check{P}_{D}-\hat{P}_{D}(0, \bar{z} ; \Delta) \quad \text { at } \quad \hat{Y}=0,
$$

and

$$
\check{W}^{(I)} \rightarrow 0, \quad \check{U}^{(I)} \rightarrow \lambda_{0} \check{Q}_{\bar{z}}^{(6)}(s, \bar{z} ; \Delta) \quad \text { as } \quad \dot{Y} \rightarrow \infty
$$

where $\check{Q}^{(m)}(s, \bar{z} ; \Delta)$ determined from (A 6$)$.

It therefore follows that $\check{W}^{(I)}$ is still given by (A 8) but with $\check{\eta}=\left(\lambda_{0} s\right)^{\frac{1}{3}} \hat{Y}$, while $\check{U}^{(I)}$ is now

$$
\begin{aligned}
\check{U}^{(I)}=\lambda_{0} \pi\left(\lambda_{0} s\right)^{-\frac{5}{3}} & \left\{\check{P}_{D_{\bar{z} \bar{z}}}\left[\mathrm{Gi}(\check{\eta})-3^{-\frac{1}{2}} \mathrm{Ai}(\check{\eta})\right]\right. \\
& \left.-3^{\frac{1}{3}} \Gamma\left(\frac{1}{3}\right)\left[\check{P}_{D_{\bar{z} \bar{z}}}+s^{2} \check{P}_{D}-s \hat{P}_{D}(0, \bar{z} ; \Delta)\right] \frac{1}{\pi} \int_{0}^{\grave{\eta}} \operatorname{Ai}(t) \mathrm{d} t\right\} .
\end{aligned}
$$

Substituting this result into (B 15) and using the asymptotic behavior of the Airy functions for large values of their arguments (Abramowitz \& Stegun 1964, pp. 448-450) shows that

$$
\left(9 \lambda_{0}^{5}\right)^{-\frac{1}{3}} \Gamma\left(\frac{1}{3}\right)\left[\check{P}_{D_{\bar{z} \bar{z}}}+s^{2} \check{P}_{D}-s \hat{P}_{D}(0, \bar{z} ; \Delta)\right]=-\check{Q}_{\bar{z}}^{(1)}(s, \bar{z} ; \Delta)
$$


and since (B 7), (B 10) and (B 12) imply that $\hat{P}_{D_{\hat{x}}}(0, \bar{z} ; \Delta)=0$, this equation can be inverted to give

$$
\left(9 \lambda_{0}^{5}\right)^{-\frac{1}{3}} \Gamma\left(\frac{1}{3}\right)\left(\hat{P}_{D_{\hat{x} \hat{x}}}+\hat{P}_{D_{\bar{z} \bar{z}}}\right)=-Q_{\bar{z}}^{(1)}(\hat{x}, \bar{z} ; \Delta)
$$

where $Q^{(m)}(\hat{x}, \bar{z} ; \Delta)$ is determined from (3.53). The solution to (B 18) that matches onto $(3.55)$ as $\hat{x} \rightarrow \infty$ is

$$
\begin{aligned}
\left(9 \lambda_{0}^{5}\right)^{-\frac{1}{3}} \Gamma\left(\frac{1}{3}\right) \hat{P}_{D}=-\frac{\bar{\beta}}{2 \pi} f_{0}^{\pi / \bar{\beta}} f_{0}^{\infty} Q^{(1)}(\xi, \zeta ; \Delta) \operatorname{Re}\{\cot \bar{\beta}[\bar{z}-\zeta+\mathrm{i}(\hat{x}-\xi)] \\
+\cot \bar{\beta}[\bar{z}-\zeta+\mathrm{i}(\hat{x}+\xi)]\} \mathrm{d} \xi \mathrm{d} \zeta
\end{aligned}
$$

where $f$ denotes the Cauchy principal value.

\section{REFERENCES}

Abramowitz, M. \& Stegun, I. A. 1964 Handbook of Mathematical Functions. US National Bureau of Standards.

Bennett, J. \& Hall, P. 1988 On the secondary instability of Taylor-Görtler vortices to Tollmien-Schlichting waves in fully developed flows. J. Fluid Mech. 186, 445-469.

BenNey, D. J. \& BERGERoN, R. F. 1969 A new class of nonlinear waves in parallel flows. Stud. Appl. Math. 48, 181-204.

Bodonyi, R. J. \& SMITh, F. T. 1981 The upper branch stability of the Blasius boundary layer including non-parallel flow effects. Proc. Roy. Soc. A 375, 65-92.

Choudhari, M., Hall, P. \& Streett, C. 1992 On the spatial evolution of longwavelength Görtler vortices governed by a viscous-inviscid interaction. Part 1: the linear case. ICASE Rep. 92-31.

CowleY, S. J. 1987 High frequency Rayleigh instability of Stokes layers. In Stability of Time Dependent and Spatially Varying Flows (ed. D. L. Dwoyer \& M. Y. Hussaini), pp. 261-275. Springer.

Ellingsen, T. \& Palm, E. 1975 Stability of linear flow. Phys. Fluids 18(4), 487-488.

Goldstein, M. E. 1976 Aeroacoustics. McGraw-Hill.

GoldSTEIN, M. E. 1994 Nonlinear interactions between oblique instability waves on nearly parallel shear flows. Phys. Fluids 6(2), 724-735.

Goldstein, M. E. \& Chог, S. W. 1989 Nonlinear evolution of interacting oblique waves on two-dimensional shear layers. J. Fluid Mech. 207, 97-120. Also Corrigendum, J. Fluid Mech. 216, 659-663.

Goldstein, M.E. \& Durbin, P.A. 1986 Nonlinear critical layers the upper branch of spatially growing Tollmien-Schlichting waves. Phys. Fluids 29, 2344-2345. 
Goldstein, M.E. \& LEE, S.S. 1992 Fully coupled resonant-triad interaction in an adverse-pressure-gradient boundary layer. J. Fluid Mech. 245, 523-551.

HaLl, P. \& Horseman, N. J. 1991 The linear inviscid secondary instability of longitudinal vortex structures in boundary layers. J. Fluid Mech. 232, 357-375.

Hall, P. \& Seddougui, S. 1989 On the onset of three-dimensionality and time dependence in Görtler vortices. J. Fluid Mech. 204, 405-420.

Hall, P. \& Sмiтh, F. T. 1991 On strongly nonlinear vortex/wave interactions in boundary-layer transition. J. Fluid Mech. 227, 641-666.

Hama, F. R. \& NuTANT, J. 1963 Detailed flow observation in the transition process in a thick boundary layer. In Proc. Heat Transfer and Fluid Mech. Inst, pp. 77-93. Stanford University Press.

HEN NINGSON, D.S. 1987 Stability of parallel inviscid shear flow with mean spanwise variation. The Aeronautical Research Institute of Sweden, Aerodynamics Department. FFA TN 1987-57.

Horseman, N.J. 1991 Some centrifugal instabilities in viscous flows. PhD thesis, Exeter University.

Hultgren, L. S. \& Gustavsson, L. H. 1981 Algebraic growth of disturbances in a laminar boundary layer. Phys. Fluids 24(6), 1000-1004.

KaCHANOV, YU.S. 1987 On the resonant nature of the breakdown of a laminar boundary layer. J. Fluid Mech. 184, 43-74.

Kachanov, Yu.S., Kozlov, V. V., Levchenko, V. Ya. \& Ramazanov, M. P. 1985 On nature of K-breakdown of a laminar boundary layer. In Laminar-Turbulent Transition (ed. V.V. Kozlov). pp. 61-74. Springer.

KaCHANOV, YU.S. \& LEVCHENKo, V. YA. 1984 The resonant interaction of disturbances at laminar-turbulent transition in a boundary layer. J. Fluid Mech. 138, 209-247.

KLebanoff, P.S. \& Tidstrom, K. D. 1959 Evolution of amplified waves leading to transition in a boundary layer with zero pressure gradient. NASA TN, D-195.

Klebanoff, P.S., Tidstrom, K.D. \& Sargent, L. M. 1962 The three-dimensional nature of boundary layer instability. J. Fluid Mech. 12, 1-34.

Kovasznay, L. S. G., Komoda, H. \& Vasudeva, B. R. 1962 Detailed flow field in transition. In Proc. Heat Transfer and Fluid Mech. Inst., pp. 1-26. Stanford University Press.

LANDAHL, M. T. 1990 On sublayer streaks. J. Fluid Mech. 212, 593-614.

LEIB, S. J. \& LEE, S. S. 1994 Nonlinear evolution of a pair of oblique instability waves in a supersonic boundary layer. Submitted to J. Fluid Mech.

MANKBADI, R. R., WU, X. \& LEE, S.S. 1993 A critical-layer analysis of the resonant triad in boundary-layer transition: nonlinear interactions. J. Fluid Mech. 256, 85-106.

Messiter, A.F. 1970 Boundary-layer flow near the trailing edge of a flat plate. SIAM J. Appl. Math. 18(1), 241-257.

NAYFEn, A.H. 1981 Effect of streamwise vortices on Tollmien-Schlichting waves. J. Fluid Mech. 107, 441-453.

NAYfeh, A.H. \& Al-MaAitah, A. 1988 Influence of streamwise vortices on TollmienSchlichting waves. Phys. Fluids. 31(12), 3543-3549. 
NishioKa, M., AsAI, M. \& IIDA, S. 1979 In Laminar-Turbulent Transition. IUTAM Mtg. Stuttgart.

Prandtl, L. 1935 Aerodynamic theory 3. p. 3. Springer.

Rozhкo, S.B. \& Ruban, A.I. 1987 Longitudinal-transverse interaction in a threedimensional boundary layer. Fluid Dyn. 22(3), 362-371.

Smith, F.T. \& Burggraf, O.R. 1985 On the development of large-sized short-scale disturbances in boundary layers. Proc. Roy. Soc. A 399, 25-55.

Stewartson, K. 1969 On the flow near the trailing edge of a flat plate II. Mathematika 16, 106-121.

STUART, J.T. 1965 The production of intense shear layers by vortex stretching and convection. NPL. Aero. Res. Rep. 1147. Also NATO AGARD Rep. 514.

Wu, X., LEE, S.S. \& COWLEY, S.J. 1993 On the weakly nonlinear three-dimensional instability of shear layers to pairs of oblique waves: the Stokes layer as a paradigm. J. Fluid Mech. 253, 681-721.

Wundrow, D. W., Hultgren, L.S. \& Goldstein, M. E. 1994 Interaction of oblique instability waves with a nonlinear plane wave. J. Fluid Mech. 264, 343-372. 


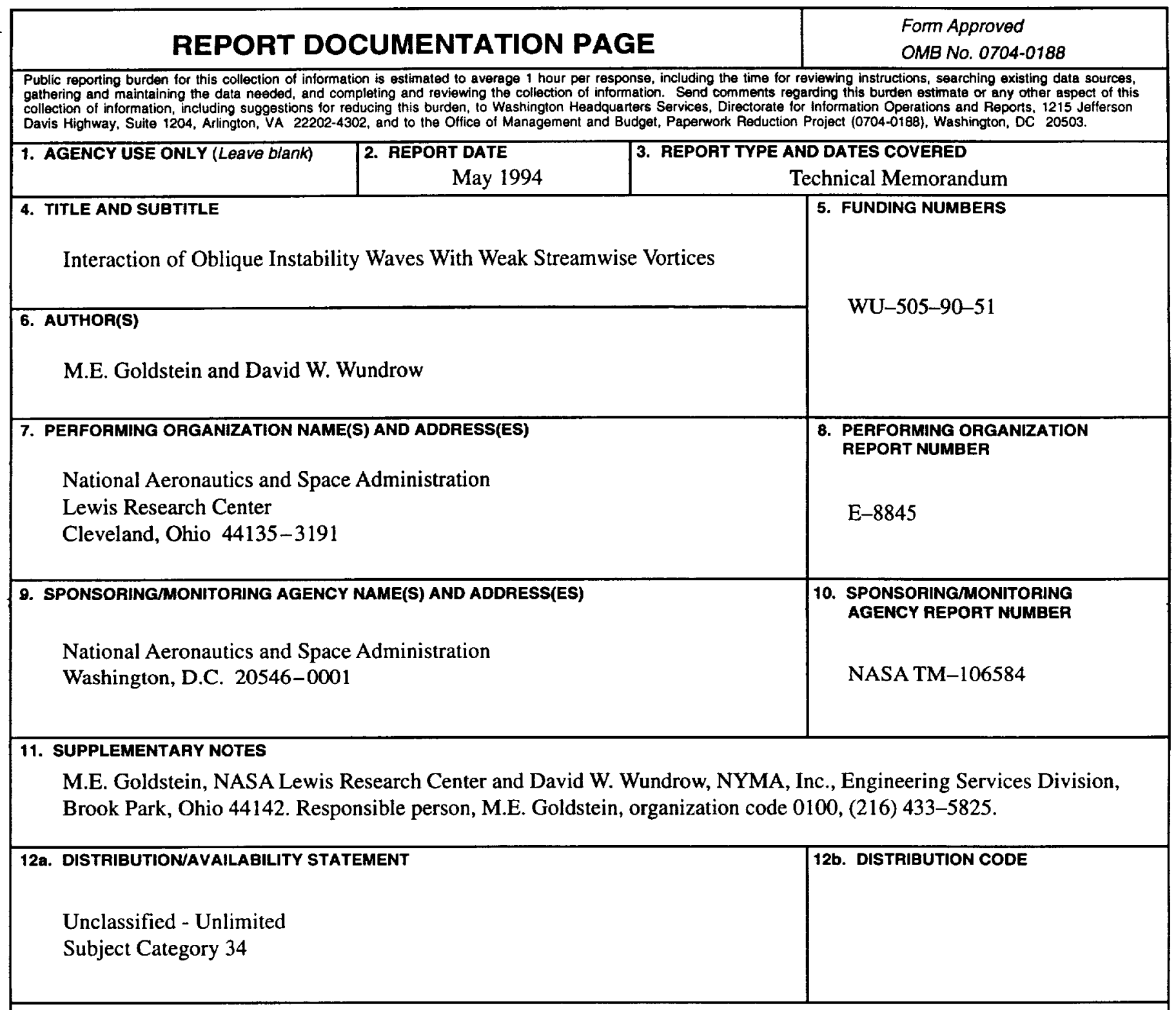

13. ABSTRACT (Maximum 200 words)

This paper is concerned with the effect of a weak spanwise-variable mean-flow distortion on the growth of oblique instability waves in a Blasius boundary layer. The streamwise component of the distortion velocity initially grows linearly with increasing streamwise distance, reaches a maximum, and eventually decays through the action of viscosity. This decay occurs slowly and allows the distortion to destabilize the Blasius flow over a relatively large streamwise region. It is shown that even relatively weak distortions can cause certain oblique Rayleigh instability waves to grow much faster than the usual two-dimensional Tollmien-Schlichting waves that would be the dominant instability modes in the absence of the distortion. The oblique instability waves can then become large enough to interact nonlinearly within a common critical layer. It is shown that the resulting nonlinearity is weak and that the common amplitude of the interacting oblique waves is governed by the amplitude evolution equation derived in Goldstein \& Choi (1989). The implications of these results for Klebanoff-type transition are discussed.

\begin{tabular}{|c|c|c|c|}
\hline \multirow{2}{*}{\multicolumn{3}{|c|}{$\begin{array}{l}\text { 14. SUBJECT TERMS } \\
\text { Transition; Instability }\end{array}$}} & \multirow{2}{*}{\begin{tabular}{|c|} 
15. NUMBEF OF PAGES \\
53 \\
16. PAICE CODE \\
A04 \\
20. \\
\end{tabular}} \\
\hline & & & \\
\hline $\begin{array}{l}\text { 17. SECURITY CLASSIFICATION } \\
\text { OF REPORT } \\
\text { Unclassified }\end{array}$ & $\begin{array}{l}\text { 18. SECURITY CLASSIFICATION } \\
\text { OF THIS PAGE } \\
\text { Unclassified }\end{array}$ & $\begin{array}{l}\text { 19. SECURITY CLASSIFICATION } \\
\text { OF ABSTRACT } \\
\text { Unclassified }\end{array}$ & 20. LIMITATION OF ABSTRACT \\
\hline 1754 & & & $\begin{array}{l}\text { andard Form } 298 \text { (Rev. 2-89) } \\
\text { sccribed by ANSI Std. Z39-18 } \\
3-102\end{array}$ \\
\hline
\end{tabular}

